AVISO

Por no disponer de los correspondientes originales informáticos, la maquetación de este artículo difiere de la del publicado en papel. Por lo demás, los contenidos no han sufrido ninguna alteración.

Artículo publicado en el fascículo $2^{\circ}$ del tomo LXV (1997) de EMERITA, pp. 221-256

Autor: María del Pilar Hualde Pascual

\title{
EOLISMOS EN JONIA: REVISIÓN DE UN PROBLEMA DE GEOGRAFÍA INTRADIALECTAL*
}

This paper discurses several forms attested in Ionic inscriptions and traditionally considered as «eolisms». Once we have established the corpus and studied each form separately, it is posible to exclude some of these forms as «eolisms». Finally, some reflexions about the real lesbisms in West Ionic are lined out.

\section{Introducción.}

Desde finales del siglo pasado y durante todo este siglo, distintos estudiosos de los dialectos griegos han venido haciendo notar que en las inscripciones ${ }^{1}$ de la Dodecápolis jonia de Asia Menor se documentan ciertas formas extrañas al dialecto jonio. Estas formas, ya en su tratamiento fonético, ya en su formación, serían supuestamente eolias, en concreto atribuíbles al lesbio,

* Una versión previa de este trabajo se leyó como comunicación al XXIII Simposio de Lingüística (Lérida, diciembre de 1993) con el título de «Eolismos en Jonia: una revisión». Agradezco a los Dres. Bernabé, García Teijeiro y de la Villa las observaciones que hicieron al trabajo en aquella ocasión.

1 La cita más frecuente de inscripciones se realiza mediante las convenciones siguientes: $B M C=$ Catalogue of the Greek Coins of Ionia by Barclay V. Head, edited by Reginald Stuart Poole, London, 1882; Dan. Mus. Ionia = Sylloge Nummorum Graecorum: The Royal Collection of Coins and Medals, Copenhagen, 1946; DGE = E. Schwyzer, Dialectorum Graecorum Exempla Epigraphica Potiora, Hildesheim, 1960 (reimpr.); EpAnat = Epigraphica Anatolica; IAIGL = M. Almagro, Las inscripciones ampuritanas, griegas, ibéricas y latinas, Barcelona, 1952; ICh = F. Graf, Nordionische Kulte, Zurich, 1985 (apéndice de inscripciones de Quíos); IEK = H. Engelmann - R. Merkelbach, Die Inschriften von Erythrai und Klazomenai, Bonn 1972-1973; IEph. = V.V. A.A., Die Inschriften von Ephesos Ia-VIII2, Bonn 1979-1984; IG = Inscriptiones Graecae; Inventaire Waddington = Inventaire sommaire de la Collection Waddington, ed. E.Babelon, Paris, 1897; Kinns = E. Kinns, Studies in the coinage of Ionia (tesis doctoral inédita), Oxford, 1980; LSCG = F. Sokolowski, Lois sacrées des cités grecques, Paris, 1969; Münsterberg = R. Münsterberg, Die Beamtennamen auf den Griechischen Münzen, Wien, 1911-1927; PP = La Parola del Passato; SEG = Supplementum Epigraphicum Graecum; SGDI = H. Collitz - F.Bechtel, Sammlung der griechische Dialektinschrifte, Götingen, 1894-1915. 
M. P. Hualde Pascual - Eolismos en Jonia: Revisión de un problema de geografía intradialectal

dialecto hablado en la Eólide, vecina de Jonia y limítrofe con algunas de las ciudades situadas más al norte de la Dodecápolis. Este hecho ha llevado en numerosas ocasiones a volver sobre el conocido pasaje de Heródoto, I 142.3-4, en el que se dividen las doce ciudades jonias en cuatro grupos según su modalidad dialectal: Una modalidad caria, que se hablaría en Mileto, Miunte y Priene; una modalidad lidia, representada por el jonio de Éfeso, Colofón, Teos, Lébedos, Clazómenas y Focea; una modalidad eolia propia del dialecto de Quíos y Eritras; por último, una modalidad «pura», hablada únicamente en Samos. No obstante, esta división herodotea en modo alguno se ve respaldada por los datos epigráficos de las ciudades en cuestión ${ }^{2}$.

Al margen del testimonio de Heródoto, el primer autor moderno que hace notar la presencia de eolismos en Jonia es F.Solmsen ${ }^{3}$, quien precisamente señala la presencia del elemento eolio en Focea, una de las ciudades que no pertenecerían a la modalidad dialectal eolia, según la división herodotea, sino a una supuesta modalidad lidia. Después del artículo de Solmsen otros autores hacen mención de lesbismos en la Dodecápolis ${ }^{4}$, tanto en manuales como en monografías o trabajos concretos referidos a la geografía dialectal del jonio. Por regla general, estos autores, sin ceñirse a la división de Heró-

2 Ya lo advierte Jeffery, en L. H. Jeffery - A. Johnston, The Local Scripts of Archaic Greece. A Study of the origin with corrections and supp. (= LSAG), Oxford, 1990, p. 327, asimismo López Eire en «En torno a la lengua del Corpus Hippocraticum», Emerita 52, 1984, p.340.

3 En su artículo «Zur Charakteristik der Mundart von Phokaia», KZ 34, 1897-1898, pp. 554-558.

4 C. D. Buck, The Greek Dialects (= Dialects), Chicago, 1955, pp.65 y 143; SGDI IV, p. 908; E. Schwyzer, Griechische Grammatik I, München, 1939, pp. 86/87; F. Bechtel, Die griechische Dialekte III (= Dialekte), Berlin, 1924, pp. 31-32; A. Thumb - A.Scherer, Handbuch der griechischen Dialekte (= Handbuch), Heidelberg, 1959, p. 245; M. Sakelariou, La migration grecque en Ionie, Athènes, 1958, pp. 288-297; K. A. Garbrah, A Grammar of the Ionic Inscriptions from Erythrae, Meisenheim am Glan, 1978, p.15; A. López Eire, «Geographie intradialectale de l'ionien-attique», Verbum 10, 1987, p. 164; A. Hernández Vázquez, «El jonio oriental», Studia Zamorensia 8, 1987, pp. 287-289; E. Sanmartí - R. A. Santiago, «Une lettre grecque sur plomb trouvée à Emporion (Fouilles 1985)», ZPE 68, p. 121; R. A. Santiago, «Epigrafía dialectal emporitana», en Dialectologica Graeca. Actas del II Coloquio Internacional de Dialectología Griega, Madrid, 1993, p. 290 y 292. Recientemente, L. Pérez Vilatela, «El plomo grecoibérico de Sagunto y el matiz eolio focense», Emerita 63, 1995, pp. 320-322 y K. Stüber, Zur dialektalen Einheit des Ostionischen, Innsbruck, 1996, pp. 82-84.

EMERITA. Revista de Lingüística y Filología Clásica (EM) - LXV 2, 1997, pp. 221-256 
M. P. Hualde Pascual - Eolismos en Jonia: Revisión de un problema de geografía intradialectal

doto, limitan los supuestos eolismos a las variedades dialectales del norte de Jonia, precisamente aquellas que, por su vecindad con la Eólide (Quíos, Eritras), o por su origen eolio (Focea, Esmirna), podrían justificar la presencia de lesbismos en su subdialecto. No obstante, hay datos que muestran que los supuestos eolismos no se restringen a la zona norte de Jonia, sino que se documentan en toda la Dodecápolis e incluso en jonio de las Cícladas, hecho que se compadece bien con la idea de que el jonio de Asia y el jonio insular no presentan diferencias que permitan establecer una variedad dialectal entre ellos ${ }^{5}$.

En primer lugar, al hablar de eolismos en jonio debemos necesariamente precisar el concepto de eolismo a partir de la llamada lingüística de los con$\operatorname{tactos}^{6}$. Dos maneras habría, al menos, para justificar la naturaleza de los eolismos, bien acudiendo al substrato, en aquellas ciudades que hayan tenido un origen lesbio (Focea, Esmirna) e interpretar los lesbismos en cuestión como vestigios lingüísticos de su pasado eolio ${ }^{7}$, bien apelando al adstrato en el resto de las ciudades, para las que deberemos más bien considerar la existencia de un área geográfica en la que se producen hechos de interinfluencia mutua entre el jonio y el eolio minorasiático, preferentemente en la zona limítrofe entre ambos territorios. Este hecho originará asimismo la presencia de abundantes jonismos en lesbio. Dado que los mencionados eolismos no limitan su aparición a las ciudades de Focea y Esmirna, sino que aparecen dispersos por un ámbito geográfico más amplio, es preferible rechazar la posibilidad de que se deban a la acción de substrato. Debemos partir más bien de hechos de adstrato, en concreto de una situación de bilingüismo que posibilite la acción mutua de un dialecto sobre otro. De esta manera, un indivi-

5 Idea defendida por A. Hernández Vázquez, art. cit., pp. 279-281.

6 La lingüística de los contactos nace en los albores del siglo XX de la mano de $\mathrm{H}$. Schuchardt, D. H. Hesseling y A. Coelho. Citaremos por su relevancia los estudios de H. Vogt, «Language Contacts», Word 10, 1954, 365-374 y U. Weinreich, Languages in Contact. Findings and Problems, Mouton, The Hague, 1963. Recientemente destaca la obra de A. Elizaincín, Dialectos en contacto (Español y portugués en España y en América), Montevideo, 1992, cuyo capítulo 2 incluye cuestiones generales de interés sobre la dialectología de los contactos.

7 Al sustrato lingüístico ha apelado recientemente Pérez Vilatela para hablar de eolismos, cf. art. cit., pp. 331-333.

EMERITA. Revista de Lingüística y Filología Clásica (EM) - LXV 2, 1997, pp. 221-256 
M. P. Hualde Pascual - Eolismos en Jonia: Revisión de un problema de geografía intradialectal

duo bilingüe, cuando hable su dialecto materno, introducirá en él rasgos del dialecto aprendido (denominados préstamos) y cuando hable el dialecto aprendido introducirá en él rasgos de su dialecto materno (denominados interferencias $)^{8}$. Esta distinción, que se ha empleado con éxito en el estudio de dialectos vivos, no es fácil de aplicar a nuestro trabajo, que se ve necesariamente limitado al estudio de formas exclusivamente epigráficas que nada nos dicen sobre factores sociales o personales del cantero que ha realizado la inscripción.

Por otra parte, estos rasgos específicos del eolio presentes en las inscripciones jonias pertenecerán tanto a la fonética y a la morfología, como al vocabulario, a la sintaxis y a la entonación y los rasgos prosódicos ${ }^{9}$. Evidente-

8 Seguimos la diferencia entre interferencia y préstamo establecida por Elizaincín, ob. cit. pp. 59-60. Estudios anteriores como el de Vogt, art. cit., p. 369 consideran el estudio de los préstamos como parte del estudio de las interferencias. Pese a las diferencias de matiz en el empleo del término 'interferencia' por parte de los distintos autores, parece haber unanimidad sobre que se trata, a diferencia de los préstamos, de un hecho inconsciente por parte del hablante y producido preferentemenete en el ámbito de la fonética. Por otra parte, hay autores que confinan los préstamos al campo léxico, sin que haya acuerdo en este punto. Una clasificación de los préstamos de distintos niveles (léxicos, morfológicos, fonéticos) en L. Deroy, L'emprunt linguistique, Paris, 1956.

9 En este sentido hay indicios en las inscripciones de que la famosa distribución de las ciudades jonias de Heródoto hace referencia a cuestiones de prosodia y entonación, es decir, de lengua hablada. Desde este punto de vista, cobra especial relevancia la presencia de la geminada dental sorda en posición inicial, documentada tras el fonema dorsal sordo $/ \mathrm{k} /$ en una

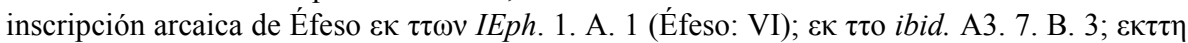

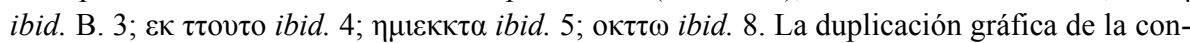
sonante se ha explicado desde hace tiempo como reflejo de un sonido de transición entre la dorsal precedente y la dental, motivado tal vez por el influjo de las lenguas autóctonas, como

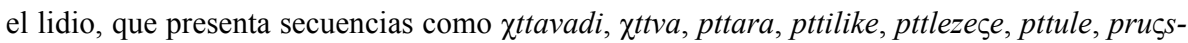
$s i$, sssveze etc. Sin poder llegar a conclusiones definitivas al respecto, por documentarse este hecho en una sola inscripción de una sola ciudad, debemos, no obstante, recordar que Éfeso es una de las ciudades para la que Heródoto defiende un tinte dialectal lidio. En este caso el texto del historiador aludiría más bien a cuestiones de pronunciación autóctona que a otro tipo de elementos lingüísticos concretos. Para esta idea cf. también Jeffery $L S A G$, p. 32: «Perhaps the speech of the first group (Myletos, Myous, Priene) was tinged with a flavour of Carian; that of second group (Ephesos, Kolophon, Lebedos, Teos, Klazomewnai, Phokaia) with Lydian; that of the third (Chios, Erythrai) with the Aiolic Greek of their northern neighbours; the purest Ionic being then that of the fourth, the island Samos».

EMERITA. Revista de Lingüística y Filología Clásica (EM) - LXV 2, 1997, pp. 221-256 
M. P. Hualde Pascual - Eolismos en Jonia: Revisión de un problema de geografía intradialectal

mente, desde el momento en que operamos exclusivamente con una lengua escrita, no podemos acceder al estudio de algunos de estos rasgos como es la entonación o la prosodia del jonio y, por otra parte, no parece claro que el hecho de que una palabra se utilice en una u otra zona dialectal pueda ser considerado como marca de dialecto ${ }^{10}$. Asimismo el estudio sintáctico de los textos epigráficos, en su mayoría breves y a veces redactados en niveles de lengua muy específicos (religioso-formular, lengua de la cancillería, etc) resulta necesariamente limitado. Así, aun a sabiendas de que las conclusiones de este trabajo serán parciales, nos limitaremos a revisar aquellos hechos fonéticos o morfológicos que, considerados como rasgos eolios en las inscripciones jonias, pudieran justificar la presencia de un elemento eolio en la Jonia minorasiática e insular, intentando calibrar, en la medida de lo posible, si estos préstamos son simples hechos esporádicos en el habla o si llegan a integrarse en el sistema del dialecto jonio. Para ello intentaremos buscar contraejemplos en la aparición de las formas jonias esperables frente al eolismo, en la misma época y preferentemente en la misma ciudad. Esta pretensión se ve en buena medida entorpecida por la escasez de inscripciones arcaicas de cada ciudad (s.VI-V) y por la temprana aparición de aticismos y de formas de koiné en Jonia.

A las dificultades mencionadas se añade que tanto en el estudio del dialecto jonio como del lesbio encontramos la dicotomía existente entre las fuentes epigráficas y las literarias. Es sabido que una lengua literaria, afectada por hechos tan diversos como la tradición, la trasmisión del texto, su propio carácter artificial, etc., no puede ponerse en el mismo nivel de fuerza testimonial que los textos epigráficos. Desde este punto de vista no consideraremos como eolismo una determinada forma por el solo hecho de que aparezca en los poetas lesbios, si su carácter eolio no se ve corroborado por su presencia en textos epigráficos dialectales. Asimismo trataremos de distinguir me-

10 Agradezco a la Dra. Hernández Vázquez de la Universidad de Salamanca la información que me envía per litteras acerca de que no parece que los rasgos léxicos sirvan para diferenciar dialectos, sino únicamente para contribuir a su diferenciación, pero siempre apoyados en rasgos de otro tipo. En opinión de la Dra. Hernández Vázquez, que ha realizado un exhaustivo estudio sobre el léxico de las inscripciones jonias, la mayor parte de los términos que presentan alguna peculiaridad léxica son arcaísmos o elecciones, lo que les resta valor como marca de dialecto.

EMERITA. Revista de Lingüística y Filología Clásica (EM) - LXV 2, 1997, pp. 221-256 
M. P. Hualde Pascual - Eolismos en Jonia: Revisión de un problema de geografía intradialectal

todológicamente si los eolismos son más o menos significativos según se trate de innovaciones, elecciónes o arcaísmos compartidos por el eolio y el jonio de Asia y de las islas.

Sentados estos principios pasamos a mencionar los hechos fonéticos y morfológicos objeto de nuestro estudio y su distribución geográfica:

1. Geminación en el contexto de primer alargamiento por compensación.

2. Resolución como diptongo del contexto de segundo alargamiento por compensación.

3. Formas con /a:/ mantenida en época arcaica.

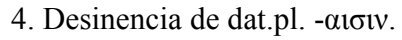

5. Contracción /a:-o/ > /a:/.

6. Palatalización de la dental sonora en contacto con yod.

7. Reducción del grupo -sthl- a -sl-.

8. Tratamiento dorsal de la labiovelar en las formas del tipo окоц, окообо, око et al.

9. Participio de perfecto flexionado con *-nt.

10. Conjugación atemática de los uerba uocalia.

11. Declinación de numerales.

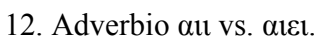

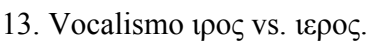

Un primer acercamiento a la distribución de los datos epigráficos pone de relieve que los supuestos eolismos de la Dodecápolis no se ciñen a la repartición presentada por Heródoto que mencionábamos al principio. Los datos de supuestos eolismos en las diversas ciudades se explicitan en el cuadro que aquí se presenta y que deja ver una relativa acumulación de datos en las ciudades más norteñas de la Dodecápolis, pero también habría presencia de rasgos eolios en ciudades situadas tan al sur como Mileto, e incluso, como dijimos, fuera de la Dodecápolis, en las islas de Tasos y Amorgos: 
M. P. Hualde Pascual - Eolismos en Jonia: Revisión de un problema de geografía intradialectal

QUI ERI CLA FOC ESM TEO COL EFE SAM PRI MIU MIL AMO TAS

\begin{tabular}{|c|c|c|c|c|c|c|c|c|c|c|c|c|c|}
\hline Geminación & + & + & - & + & - & + & - & - & + & - & - & - & - \\
\hline Diptongación & + & - & - & + & - & - & - & - & - & - & - & + & - \\
\hline /a:/ & + & + & - & + & - & - & - & - & + & - & - & - & - \\
\hline$-\alpha ı \sigma ı v$ & - & - & - & + & - & - & - & - & - & - & - & - & - \\
\hline /a: $-\mathrm{o} />/ \mathrm{a}: /$ & - & + & - & - & - & - & - & - & - & - & - & - & - \\
\hline$\delta 1>\zeta_{1}$ & - & - & - & + & - & - & - & - & - & - & - & - & - \\
\hline -sthl- > -sl- & + & + & - & - & - & - & - & - & - & - & - & - & + \\
\hline око- & - & + & - & + & - & - & - & - & - & - & - & - & - \\
\hline$\gamma \varepsilon \gamma \omega v \varepsilon \sigma \nu \tau \varepsilon \varsigma$ & + & - & - & - & - & - & - & - & - & - & - & - & - \\
\hline$\delta \iota \psi \alpha \nu \tau \imath$ & - & - & - & + & - & - & - & - & - & - & - & - & - \\
\hline Decl. numer. & + & - & - & - & - & - & - & - & + & - & - & - & - \\
\hline$\alpha u$ & + & - & - & - & - & - & - & - & - & - & - & + & - \\
\hline ipos & + & + & - & + & - & - & - & - & + & - & - & - & - \\
\hline
\end{tabular}

(QUI, Quíos; ERI, Eritras; CLA, Clazómenas; FOC, Focea; ESM, Esmirna; TEO, Teos; COL, Colofón; EFE, Éfeso; SAM, Samos; PRI, Priene; MIU, Miunte; MIL, Mileto; AMO, Amorgos; TAS, Tasos).

\section{Revisión y discusión de los datos.}

\subsection{Geminación en el contexto de primer alargamiento compensatorio.}

En jonio de Asia Menor se documenta con regularidad el primer alargamiento por compensación desde los testimonios más arcaicos, tanto en las ciudades más próximas a la Eólide (Eritras, Quíos), como en las que están más alejadas de esta zona. No obstante, en el dialecto de las ciudades de la Jonia del norte hay ejemplos que sugieren la posibilidad de un tratamiento alternativo para la resolución del contexto de primer alargamiento, la geminación ${ }^{11}$, que representaría un arcaísmo en estas

11 Así se ha defendido desde antiguo, cf. Buck, Dialects, pp. 65 y 143; SGDI IV, p. 908; EMERITA. Revista de Lingüística y Filología Clásica (EM) - LXV 2, 1997, pp. 221-256 
M. P. Hualde Pascual - Eolismos en Jonia: Revisión de un problema de geografía intradialectal

ciudades. Es destacable el hecho de que esta geminación se encuentra restringida a los siguientes casos:

1.1.1. Dos series de antropónimos, en $\Phi \alpha v v 0^{\circ}$ y $\Delta \mathrm{vvvv}^{\circ}$ : Algunos de ellos son hipocorísticos y nombres abreviados, cuya naturaleza habrá que tener en cuenta a la hora de la interpretación de los hechos. A éstos se añade, fuera de la Dodecápolis, la forma de Tasos $\Lambda \alpha \lambda \lambda \eta \varsigma$ de difícil interpretación.

(a) Los antropónimos en $\Phi \alpha v v 0^{\circ}$ están documentados básicamente en Eritras:

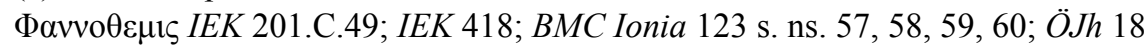

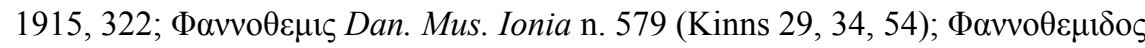
Kinns n. 319 (Eritras: 133/130). Para su formación se ha propuesto un primer elemento creado a partir de una forma básica ${ }^{*} p^{h} a$,esno ${ }^{12}$, correspondiente al

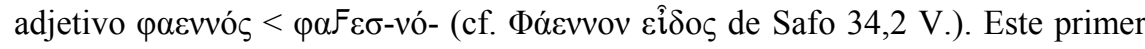

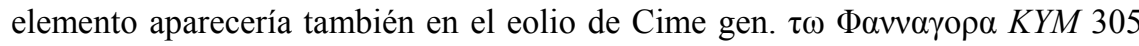
(s.II) ${ }^{13}$, forma cuyo tema originario, $\Phi \alpha \varepsilon v-$, en composición habría contraído en $\Phi \alpha v v-$, y que tendría un paralelo sin contraer, dentro de la propia Eólide en

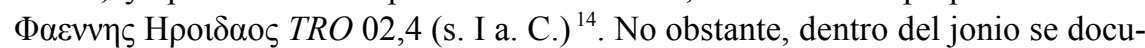
mentan, como contraejemplo, formas paralelas sin geminación en la propia ciudad de Eritras, así $\Phi \alpha v o \pi 0 \lambda ı \delta$ os EpAnat 8, 1986, p.17.26 (Eritras: V/IV), y en

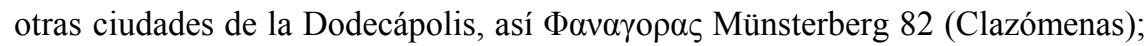

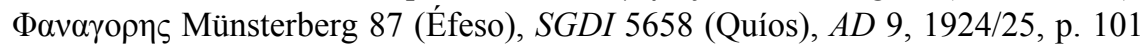

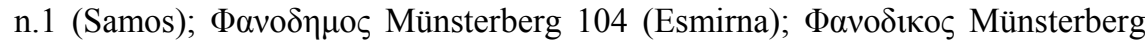
98 (Mileto); $Ф \alpha v o \pi \mathrm{o} \lambda_{1 \zeta} S E G$ XIX 580.28 (Quíos: IV/III) et al. Junto a esto se documentan dos nuevos datos de geminación en esta serie de antropónimos en ciudades de la Dodecápolis sorprendentemente alejadas del área de supuesta influencia eolia, Teos y Samos: en Teos aparece una forma $\Phi \alpha v v o \theta \varepsilon \mu 1 \varsigma$ SEG XXIX

Bechtel, Dialekte, pp. 31-32; Thumb - Scherer, Handbuch, p. 245; Sakellariou, ob. cit. p. 289-291, K. A. Garbrah, ob. cit. p. 15; López Eire, art. cit. p. 164.

12 Para esta idea cf. Bechtel, Dialekte III, p. 32; Thumb-Scherer Handbuch p. 255. 95; Garbrah, ob. cit. pp. 86-87; López Eire, art. cit. 164. Asimismo Bechtel, Die historische Personennamen des Griechischen bis zur Kaiserzeit, Hildesheim, 1964 (=HPN), p. 440 pone en paralelo la forma $\Phi \alpha v v \alpha \gamma o \rho \alpha \varsigma$ del eolio de Cime con Eritras $\Phi \alpha v v o \theta \varepsilon \mu 1$. Hay formas más

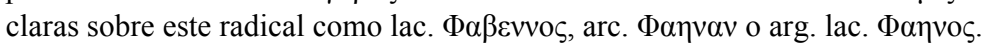

13 La cita de inscripciones de la Eólide se realiza de acuerdo a las convenciones de R. Hodot, Le dialecte éolien d'Asie. La langue des inscriptions VII a. C. - IV p. C., Paris, 1990.

14 En idea de R. Hodot, ob. cit., p. 87 y n. 224. Asimismo cree este autor que la forma

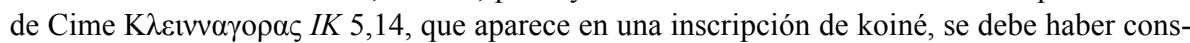

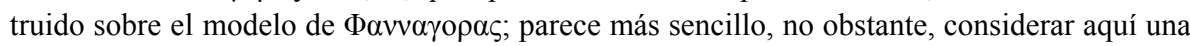
geminación expresiva en frontera de compuesto de un antropónimo.

EMERITA. Revista de Lingüística y Filología Clásica (EM) - LXV 2, 1997, pp. 221-256 
M. P. Hualde Pascual - Eolismos en Jonia: Revisión de un problema de geografía intradialectal

1149 (Teos:III/II), en un texto de difícil lectura ${ }^{15}$, que supondría un claro correlato de las formas de Eritras; un caso parecido lo constituye la forma samia $\Phi \alpha v v o \varsigma$ Münsterberg 111. A esto se une la aparición de formas similares con geminada fuera de Jonia y de la Eólide, como es el caso de los antropónimos laconios

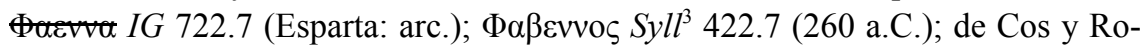

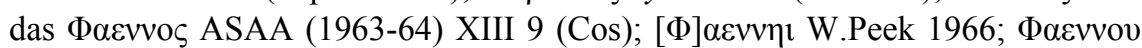
IG 12,3 n 263 (Rodas:s.II-I) ${ }^{16}$ y en estos casos la posibilidad de eolismo en laconio o rodio está evidentemente descartada. Hemos de tener en cuenta que todas estas formas, por el hecho de ser antropónimos, en ningún caso pueden tener la misma fuerza testimonial para defender la posibilidad de eolismo que la que tendría un hecho que afecte a la fonética o a la morfología fuera de la onomástica. El hecho de que estos términos sean nombres de persona permite al menos dos explicaciones para la presencia de la geminada: En primer lugar, los antropónimos están sometidos frecuentemente a las presiones de la moda; en este sentido no es de extrañar que un determinado dialecto adopte formas de otro, en este caso el lesbio, en sus nombres de persona, de la misma manera, por poner un ejemplo, que en nuestra sociedad actual hay un cierto gusto por los nombres anglosajones. En segundo lugar, todas las formas mencionadas pueden ser atribuibles a un primer elemento $\Phi \alpha v 0^{\circ}$, con geminación expresiva en frontera de compuesto o en hipocorístico ${ }^{17}$, al que pertenecerían formas de dentro y fuera de la Dodecápolis

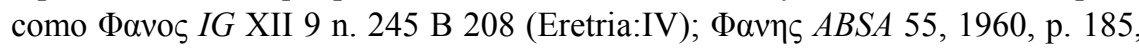
n. A I 48 (Quíos); Фavms IG II 210232 (Samos) et. al. Bien es verdad que, al no aparecer ninguna de estas formas en textos métricos, no podemos saber si la /a/ es larga (procedente de $\Phi \alpha v o-<\Phi \alpha \varepsilon v o-<\Phi \alpha F \varepsilon \sigma v o-)$ o breve ${ }^{18}$ (procedente de primer elemento $\Phi \alpha v 0^{\circ}$ ), pero, a la vista de los paralelismos como $\Phi \alpha v v o \theta \varepsilon \mu \iota \varsigma$ y

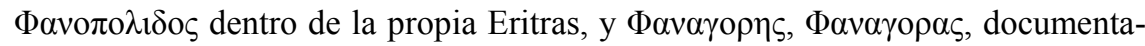
dos en Éfeso y Clazómenas, y $\Phi \alpha v v \alpha \gamma o \rho \alpha \varsigma$ en eolio de Cime, nos inclinamos a

15 El SEG recoge tanto la lectura de R. Bernhardt, $\Phi$ $\lambda \lambda_{0} \theta \varepsilon \mu 1 \varsigma$, como la de Ch. Irmer, $\Phi \alpha \nu v o \theta \varepsilon \mu 1 \varsigma$.

16 Agradezco a la doctora Striano de la Universidad Autónoma de Madrid la documentación que me ha proporcionado sobre antropónimos laconios y rodios, así como la atención que ha dedicado a comentar diversos aspectos de este estudio. Agradezco asimismo a los doctores Crespo y Maquieira de esta misma Universidad la amabilidad con que han leído este trabajo y sus valiosas sugerencias.

17 Así lo define O. Masson, «Gémination expressives dans l'anthroponymie grecque», BSL 81,1986, pp. 217-229.

18 Independientemente del origen de la /a/ breve, para lo cual cf. Chantraine, DELG, s.v. $\Phi \alpha \varepsilon .-$.

EMERITA. Revista de Lingüística y Filología Clásica (EM) - LXV 2, 1997, pp. 221-256 
M. P. Hualde Pascual - Eolismos en Jonia: Revisión de un problema de geografía intradialectal

pensar, aunque sea imposible asegurarlo, que nos encontramos ante un mismo primer término de compuesto.

(b) La segunda serie de antropónimos para los que se ha defendido la supuesta geminación eolia la componen las formas en $\Delta \mathrm{wovvv}^{\circ}$ y sus variantes $\Delta \mathrm{v} v v v^{\circ}$ y $\Delta \varepsilon \mathrm{ovvv}^{\circ}{ }^{19}$, documentadas en tres de las ciudades de la Jonia del norte, Eritras,

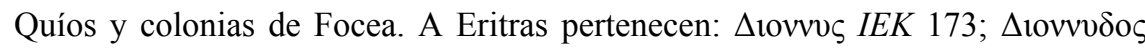
Dan. Mus. Ionia 689; BMC 133 n. 144; Münsterberg NZ 60, 1927, 69 addendum

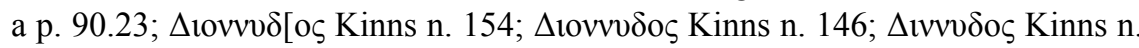
144. En Quíos la geminación aparece sólo en un antropónimo, $\Delta \mathrm{vvvv \varsigma ,} \mathrm{enorme-}$ mente productivo en la onomástica de la ciudad: $\triangle \mathrm{tvvv \varsigma SEG} \mathrm{XV} 538$ (Quíos:III); $\Delta \mathrm{v}$ ] voos SGDI 5660.3 (Quíos: s.d.); $\Delta \mathrm{v}$ ]vvos ICh. 77.2/3 (Quíos:ca. 200); $\Delta$ vvvvos EpAnat 9 p. 65 n.13.6 (Quíos: ep.rom.) ${ }^{20}$. En las colonias occidentales de Focea aparece $\Delta$ lovvis $^{21} P P 73,1981$, p. 363 (Islas Hyères, I a.C./I d.C.), junto a la forma esperable $\Delta$ iovos ib. ${ }^{22}$.

La etimología de este antropónimo ha suscitado asimismo no poca controversia: la opinión más generalizada es que este primer término de compuesto se relaciona con el nombre de Dioniso ${ }^{23}$. Así las formas de Eritras y Focea $\Delta 10 v v v \delta o \zeta$, $\Delta$ lovvis estarían creadas sobre el nombre de Dioniso a partir de una forma básica *di,os-nusos, con la forma de genitivo del nombre de Zeus como primer término de compuesto.

Por su parte las formas en $\Delta \mathrm{vvvv}^{\circ}$, si realmente fueran variantes del nombre

19 La identificación de las tres formas está generalmente reconocida cf. M. S. Ruipérez, «The Mycenaean name of Dionisos» Res Mycenaea, 1983, pp. 408-412; O. Masson, «Monetary magistrates in Abdera and Maroneia (V/IV a. C. )» RN 26, 1984, p. 51.

20 Junto a la forma con geminada aparece en esta inscripción la forma habitual del nom-

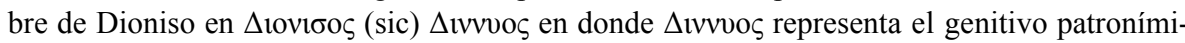
co.

${ }^{21}$ Nótese la grafía $<\mathrm{I}>$ por $<\mathrm{Y}>$, tal vez por error del escriba, y prueba de la anteriorización $/ \mathrm{u} />/ \ddot{\mathrm{u}} /$.

22 Sobre la presencia masaliota en un santuario del dios Aristeo en el archipiélago de las Islas Hyères (costa de la Provenza francesa), cerca de Olbia, y el estado de las excavaciones cf. J. Coupry - M. Giffault «La clientèle d'un sanctuaire d'Aristée aux îles d'Hyères», PP 73, 1982, pp. 360-361.

23 O. Masson, art. cit. en n. 17, pp. 48-60, considera un hecho la relación de estos hipocorísticos con el nombre de Dioniso, dando la razón a Hoffmann Gr. Dial. II pp. 262-263.; asimismo Robert, que en «Quelques noms de personnes grecs», Ant. Class. 32, 1963, 5-17, hace un detallado estudio de la forma de hipocorístico $\Delta$ tovv $\varsigma$ y sus variantes.

EMERITA. Revista de Lingüística y Filología Clásica (EM) - LXV 2, 1997, pp. 221-256 
M. P. Hualde Pascual - Eolismos en Jonia: Revisión de un problema de geografía intradialectal

de Dioniso, tendrían correlato en la forma tardía de la Eólide minorasiática $\triangle$ ıvvvбos SEG XXXXII 1243 de Cime, a partir de una etimología popular para el nombre del dios *d,is-nusos 'dos veces niño', que haría referencia al doble nacimiento del dios, del vientre de su madre y del muslo de Zeus ${ }^{24}$, con el tratamiento de geminación eolio para el grupo -sn-. No obstante, la relación de este antropónimo con el nombre del dios no es segura, ya que pueden pertenecer a un compuesto en $\Delta \mathrm{v} v(v)^{\circ}$ de *d,isnós 'temible' ${ }^{25}$, y en cualquier caso, al estar tratando con nombres de persona, se puede aplicar el mismo razonamiento que en el punto anterior, bien pueden deber su geminada a que son nombres lesbios impuestos en jonio por una cuestión de moda, bien pueden presentar simplemente la geminación expresiva propia de los hipocorísticos.

(c) Finalmente, encontramos la forma del jonio insular $\Lambda \alpha \lambda \lambda \eta \varsigma$ A $\rho \tau i \lambda \varepsilon \omega I G$ XII 8.277.14 (Tasos: s. VI/V). En el antropónimo $\Lambda \alpha \lambda \lambda \eta \varsigma$ se ha pretendido $\operatorname{ver}^{26}$ un segundo término $-\alpha \lambda \lambda \eta \varsigma$, contracción de $-\alpha F \varepsilon \lambda \lambda \eta \varsigma$, hom. $\dot{\alpha} \varepsilon \lambda \lambda \eta$ ' $I l$. III,13, jon. $\dot{\alpha} \lambda \eta \varsigma^{\prime}$ 'reunido', $(<* \alpha-F \varepsilon \lambda-v \eta \varsigma)$. Esto presupone que la forma tendría un doble carácter jonio-eolio: eolio en su tratamiento de la secuencia -ln- con geminación $\dot{\alpha} \varepsilon \lambda v \eta \eta \varsigma>\dot{\alpha} \varepsilon \lambda \lambda \eta \dot{\eta} \varsigma$, y jonia en cuanto a su vocalismo /a:/ (cf. jon. $\dot{\alpha} \lambda \eta \varsigma$ Hdt., Hp., Call., Hes.), posiblemente procedente de $\dot{\alpha} \varepsilon \lambda-v \eta \varsigma^{27}$, frente a formas del lesbio literario como $\dot{\alpha} 0 \lambda \lambda \eta \dot{\zeta}$ ( $\dot{\alpha}$ ó $\lambda \varepsilon \varepsilon \varepsilon \varsigma$ Alc. 163 P., Sa., pero también Od. XIV,432, Il.

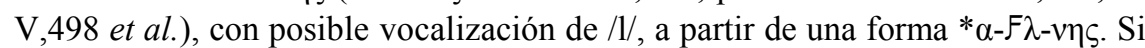
este planteamiento fuera correcto, nos encontraríamos ante una forma haplográfica, con un primer elemento $\Lambda \alpha F^{\circ}$, creado sobre *lā,os: $\Lambda \alpha F_{-}(0)-\alpha F_{\varepsilon} \lambda \lambda \eta \uparrow$, con haplografía $\Lambda \alpha \lambda \lambda \eta \varsigma^{28}$. Los problemas que presenta esta interpretación son varios: En primer lugar en jonio sería esperable un primer elemento $\Lambda \varepsilon \omega-($ de *lā,o, por metátesis de cantidad) ${ }^{29}$, en segundo lugar, no coincide el supuesto segundo tér

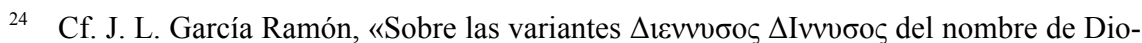
niso», en Studies in Mycenaean and Classical Greek presented to J. Chadwick, Minos, 1987, pp. 197-198.

25 Cf. García Ramón, art. cit. p. 193 n. 33, quien, en su argumentación sobre la etimología del nombre de Dioniso y sus variantes, prefiere prescindir de los antropónimos de Quíos, por no considerar suficientemente probada la pertenencia a la misma etimología.

26 F. Bechtel, $H P N$ p. 38, cree ver una forma mixta lesbio-jonia.

27 Cf. Chantraine, $D E L G$, s.v. $\dot{\alpha} \lambda \hat{\eta} \varsigma$ : «toutefois l'ionien $\dot{\alpha} \lambda \eta \dot{s}$ peut reposer aussi sur $*_{\alpha-}-F \varepsilon \lambda-v \eta \varsigma »$.

28 Esta idea en Bechtel, HPN, p. 38; Knitl, Die Sprache der ionischen Kykladen nach den inschriftlichen Quellen, München, 1938, p. 65.

29 Siempre que no entendamos también en el primer término del compuesto una contracción eolia de $\Lambda \alpha o-$ en $\Lambda \alpha$-, como las que interpretan por Garbrah op. cit. pp. 15, 43, 145 y

EMERITA. Revista de Lingüística y Filología Clásica (EM) - LXV 2, 1997, pp. 221-256 
M. P. Hualde Pascual - Eolismos en Jonia: Revisión de un problema de geografía intradialectal

mino del compuesto $-\alpha \mathcal{F}_{\varepsilon} \lambda \lambda \eta \varsigma$ con el vocalismo del eolio (literario), $\dot{\alpha} 0 \lambda \lambda \eta \dot{\zeta} \varsigma, \mathrm{y}$, además, no se conocen formas similares en lesbio epigráfico; finalmente, se añade a los obstáculos la propia complicación del recurso a la explicación haplográfica ${ }^{30}$. Por otra parte, formas de antropónimo en $\Lambda \alpha \lambda$ - y $\Lambda \alpha \lambda \lambda$ - no son desconocidas entre los nombres indígenas de Asia ${ }^{31}$. Así, el antropónimo que nos ocupa podría estar creado sobre un radical de origen minorasiático y presentar el sufijo de hipocorístico - $\varepsilon \alpha \varsigma$, jonio $-\hat{\eta} \varsigma$, muy frecuente en jonio de Asia y de las islas ${ }^{32}$. Obraría en apoyo de esta opinión la presencia, ya fuera de Jonia, en Rodas, del hipocorístico $\Lambda \alpha \lambda \lambda \hat{\alpha} \varsigma$ Rev. Bibl., NS 14, 1917, p. 293 n. 21 (ep. hel.), que puede entenderse como creado sobre la misma forma que el antropónimo de Tasos, pero con distinto sufijo de hipocorístico, así $\Lambda \alpha \lambda \lambda \eta \hat{\varsigma}$ vs. $\Lambda \alpha \lambda \lambda \hat{\alpha} \varsigma$. No parece, pues, imprescindible la interpretación como eolismo del antropónimo $\Lambda \alpha \lambda \lambda \eta$ s.

\subsubsection{Datos en textos literarios:}

A estos datos de supuesta geminación se añadirían dos nuevos datos marginales, documentados en textos literarios:

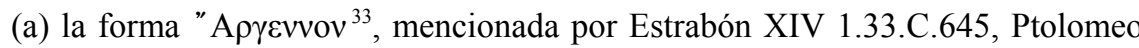
V,2,7, y Polibio XVI,8, nombre de un cabo situado en Eritras, sin que se documente mención epigráfica alguna;

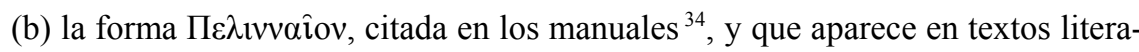
rios como nombre de una ciudad tesalia (Pi. Pyth. 10.7) ${ }^{35}$, y como orónimo de

López Eire, art. cit., p. 165 en los antropónimos $\Lambda \alpha \mu \varepsilon \delta \omega v, \Lambda \alpha \pi \rho \varepsilon \pi \eta \varsigma$, cf. infra 2. 4.

30 Aunque ciertamente la haplografía de los nombres en $\Lambda \varepsilon \omega$ - es habitual cuando el segundo término tiene inicio vocálico cf. $\Lambda \varepsilon \alpha \gamma o \rho \eta \varsigma ~ I G$ XII 8.274A.11 (Tasos, IV); $\Lambda \varepsilon \alpha \rho \varepsilon \tau \eta ı ~ I G$ XII 8.398 (Tasos, V) (cf. Knitl ob. cit. p. 74) y $\Lambda \varepsilon \alpha v \alpha \chi$ M 122.I.35 (cf. Scherer, Zur Laut- und Formenlehre der milesischen Inschriften, Diss., München, 1934, p. 26.

31 Cf. L. Zgusta, Kleinasiatische Personennamen, Prag, 1964, pp. 265-267.

32 Así lo recoge Frazer-Mathews, A Greek Lexicon of Personal Names (Andros, Delos, Rhodas, Samos) I, Oxford, 1987, p. 281: $\Lambda \alpha \lambda \lambda \hat{\eta}$.

33 Considerada eolismo por Sakellariou, op. cit., p. 291. El texto de Estrabón es el si-

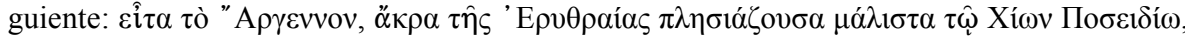

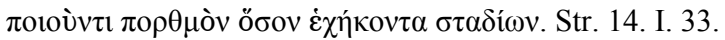

34 Buck, Dialects p. 143; Bechtel, Dialekte III, p. 138; SGDI III, 2 p. VII; Sakellariou, op. cit. p. 289.

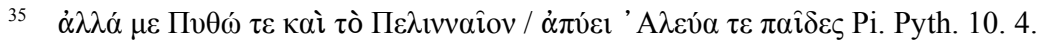

EMERITA. Revista de Lingüística y Filología Clásica (EM) - LXV 2, 1997, pp. 221-256 
M. P. Hualde Pascual - Eolismos en Jonia: Revisión de un problema de geografía intradialectal

Quíos (Ael. N.A. XVI 39, Str. XIV 1.33) ${ }^{36}$, pero cuya única mención epigráfica

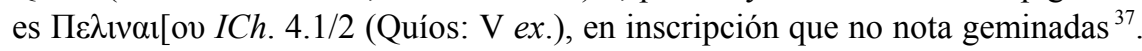
No consideramos que estos dos datos se puedan tomar como prueba definitiva de geminación eolia residual en las ciudades del norte de Jonia, puesto que en ambos términos la geminación se documenta únicamente en textos literarios ${ }^{38}$.

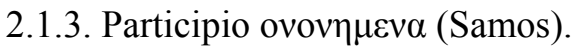

También se ha pretendido ver un caso de geminación eolia en el partici-

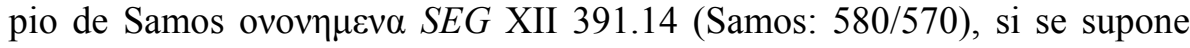

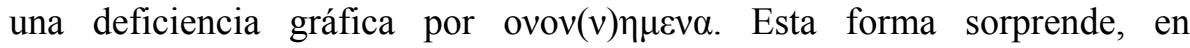
principio, porque presenta una reduplicación anómala, en un radical donde no sería esperable la reduplicación ática, ya que, según parece claro por su significado en la inscripción -evidentemente 'compradas'-, no es otra cosa que el participio pasivo de §véo $\mu \alpha 1$. En este radical, que conlleva una *,- ini-

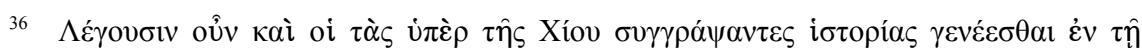

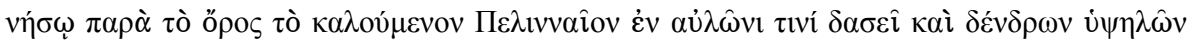

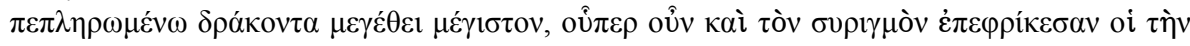

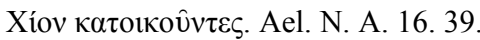

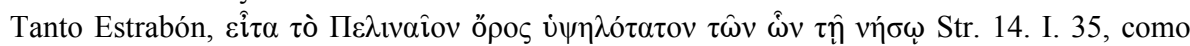

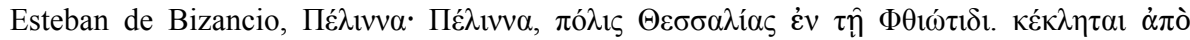

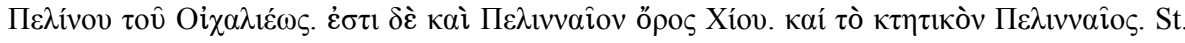
B. s. v., relacionan el orónimo de Quíos con la ciudad de Pelinna en la Ftiótide.

37 No supone problema la ausencia de notación de la geminada, puesto que en el mismo texto encontramos $\alpha \lambda(\lambda) \omega \mathrm{i}$ ib. 11. Mayor problema plantea el hecho de que en la inscripción Пє $\lambda$ ivalos designa no el monte mencionado, sino que es la epiclesis de una divinidad, posiblemente Zeus, si bien en el texto se documenta únicamente la epiclesis, pero falta el nombre del dios. Sobre los problemas para la identificación de Zeus Pelinnaios y una posible divinidad autóctona Pelinnaios, desde un punto de vista sincrónico y diacrónico, cf. F. Graf, Nordionische Kulte, Zurich, 1985 pags. 37-43. El hecho de que el monte Pelinnaios esté situado en el norte de la isla y que, por el contrario, la inscripción haya aparecido entre las ruinas de una casa de Mesa Rhamni, en el oeste de la ciudad de Quíos, y su procedencia última no sea clara, dificultan la cuestión de la vinculación entre la divinidad y el monte Pelinnaios.

38 Contra Sakellariou, op. cit., pp. 289-290, quien considera que la relación de este orónimo con Pelinna, en Tesalia, es la prueba fehaciente de su condición de eolismo. Consideramos, por el contrario, que, mientras no haya disponible documentación epigráfica de la geminación en este término, no se puede constatar su condición de eolismo.

EMERITA. Revista de Lingüística y Filología Clásica (EM) - LXV 2, 1997, pp. 221-256 
M. P. Hualde Pascual - Eolismos en Jonia: Revisión de un problema de geografía intradialectal

cial, sería esperable una forma de perfecto del tipo $\dot{\varepsilon} \omega v \eta-{ }^{39}$, tal como aparece en jonio de Quíos $\varepsilon \omega v \eta[v \tau \alpha l$ ICh. 5.16 (Quíos: 400-350), o en una forma helenística de Halicarnaso $\varepsilon \omega v \eta \mu \varepsilon v \eta$ SEG XVI.645.4 (Halicarnaso: ep. hel.) cf. át. $\dot{\varepsilon} \omega ́ v \eta \mu \alpha r$ (Aristoph.). Un segundo aspecto que sorprende en la forma ovovๆ $\mu \varepsilon v \alpha$ es la $<_{0}>$ de su segunda sílaba, frente a una pretendida reduplicación ática, según la cual sería esperable una vocal larga abierta en dicha

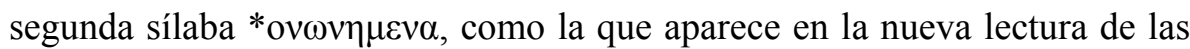
formas de Ampurias ov ov $\omega v \eta \sigma[\theta \alpha 1] S E G$ XXXIX 1088.4 (Ampurias ca. 450) ${ }^{40}$. Desde el momento de la primera publicación de la inscripción de $\operatorname{Samos}^{41}$ la interpretación de la forma que nos ocupa ha sido tema controvertido: Las primeras interpretaciones abogaban por una asimilación de la segunda vocal de timbre -o- a la pri-

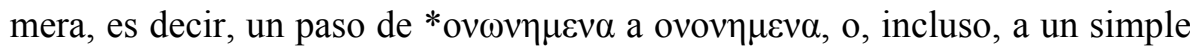
error del escriba ${ }^{42}$, que debe considerarse únicamente como posibilidad extrema, si no existe otra explicación posible del hecho en cuestión. La posibilidad de asimilación /o/-/ :/ > /o/-/o/, es decir, una asimilación de cantidad, carece de paralelos conocidos y parece, por tanto, difícilmente admisible.

Posteriormente se apuntó una posibilidad sin duda interesante y pertinente para este trabajo: considerar la forma ovovๆ $\mu \varepsilon v \alpha$ como un eolismo ${ }^{43}$, no tanto en el subdialecto de Samos como en el de su colonia Perinto ${ }^{44}$, inter

39 La presencia de esta reduplicación en un tema con *,- inicial puede explicarse si entendemos una forma analógica, creada sobre los temas en los que la reduplicación es antigua y fonética, procedente del tratamiento de la laringal, según el esquema HRe-HC cf. ${ }^{*} \mathrm{H}_{3} \mathrm{le}-\mathrm{H}_{3} \mathrm{l}-\mathrm{o} \lambda \omega \lambda$-. Paralelos en este mismo sentido se encuentran en el perfecto $\dot{\varepsilon} \mu \eta \dot{\mu} \mu \varepsilon \kappa \alpha$ Luc. Lex. 21, Ael. NA 17. 37, correspondiente a un presente $\dot{\varepsilon} \mu \varepsilon \dot{\varepsilon} \omega$, de cuyo inicial en *u- no cabe duda a la vista de ai. vámiti, y en la forma ó át. غ̇ó $\alpha \kappa \alpha$ Ar., en su forma esperable); ello hablaría a favor de una reduplicación «reciente».

40 La nueva lectura corresponde a R. A. Santiago Alvarez per litteras, frente a las lecturas previas recogidas en el SEG: ] $\alpha \vartheta \eta \eta \imath$ ov $\omega v \eta \sigma \theta \alpha \iota$ B $\alpha \sigma \pi \varepsilon \delta[$ SEG XXXVII 838. 4; ]ov ov $\sigma[\alpha \iota$ SEG XXXIX 10888. 4.

${ }^{41}$ G. Klaffenbach, «Archaische Weihinschrift aus Samos», Mitteilungen des deutscher archäologischen Instituts 6, 1953, pp. 15-20.

42 Klaffenbach, art. cit. p. 20.

43 Schwyzer, Gr. Gr. I, p. 86; Bechtel, Dialekte, I, pp. 37,59; Thumb-Scherer, Handbuch, II p. 280.

44 Desde el momento en que la inscripción es una dedicación de los perintios a la Hera

EMERITA. Revista de Lingüística y Filología Clásica (EM) - LXV 2, 1997, pp. 221-256 
M. P. Hualde Pascual - Eolismos en Jonia: Revisión de un problema de geografía intradialectal

pretación que se vería respaldada por la aparición en el mismo texto de formas flexivas para los numerales cf. $\delta v \omega \delta \varepsilon \kappa \omega v$ ib. $16 / 17^{45}$, en paralelo a las formas documentadas en la inscripción de Lofitis en Quíos ${ }^{46}$. La forma en cuestión sería, en este caso, el participio perfecto pasivo de un verbo *ỏvvéo $\mu \alpha$, que sería el correlato de la forma epigráfica del lesbio ovva 'contrato' IG Suppl. 136.b.17.21 (de *,osnā, con la geminación propia del lesbio).

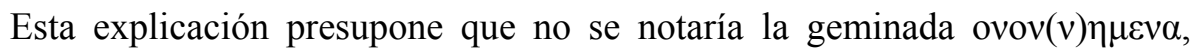
hecho por otra parte frecuente en textos epigráficos arcaicos ${ }^{47}$. En cualquier caso, la diferencia de cantidad vocálica entre las formas eolias ovvo- y del jónico-ático $\omega v$ - resulta difícil de reducir a una sola forma básica ${ }^{48}$. Dos posibilidades se ofrecen: (a) Partir de una forma previa *,!sn- a partir de la cual el alargamiento compensatorio tendría un resultado de vocal abierta /O:/ en lugar de vocal larga cerrada /o:/49 jon.át. §vo srente a lesb. ovv $\alpha$; ello implicaría admitir la posibilidad de un doble tratamiento para la secuencia oNs (1)

de Samos, el texto, en sentido estricto, nos ilustra más sobre la variedad del jonio de la colonia que del de la metrópoli.

45 D. A. Ringe, «Ionic ovovๆ $\mu \varepsilon v \alpha »$ Glotta 62, 1984,p. 47, considera que no debe ponerse en pie de igualdad las posibilidades de préstamo «eolio» en los nombres propios y en las formas desinenciales que en uno de los verbos básicos del jonio. Sin embargo es fácil que se haya producido un préstamo léxico (y fonético a la vez, con sustitución del alargamiento por la geminación), uno de los tipos de préstamo más frecuentes en situaciones de lenguas en contacto. Esto es muy probable al ser un verbo tan frecuente en el léxico del comercio, lo que parece idóneo para que en él se produzca la influencia lingüística entre zonas que tendrían necesariamente trato comercial entre ellas.

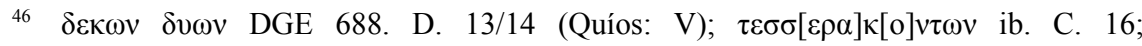
$\pi \varepsilon v \tau \eta \kappa o v \tau \omega \nu$ ib. C. 6/7; $\varepsilon v \varepsilon v \eta \kappa o v \tau \omega v$ ib. C. 26. Salvo los datos de esta inscripción de Quíos y la forma $\delta v \omega \delta \varepsilon \kappa \omega v$ de la inscripción que nos ocupa, el resto de las formas de numerales de textos epigráficos de la Dodecápolis de Asia Menor presenta la forma indeclinable habitual, desde los más antiguos testimonios; para su discusión cf. infra. .

47 Otros casos de ausencia de notación de la geminada en Samos son A 5. 2 (Samos: $575 / 550$ ); $\tau \omega \pi \mathrm{o} \lambda \omega v$ LSAG n. 17. 2 (Dedicación de un samio en Delfos: ca. 479?) (Datos tomados de nuestra tesis doctoral, El dialecto jonio de la Dodecápolis. Fonética y Morfología. Estudio intradialectal. Madrid, Universidad Autónoma, 1993. )

48 Esta idea en Ringe, art. cit. p. 47.

49 La posibilidad de que en las secuencias osn- y oms- el alargamiento sea /O: / aparece

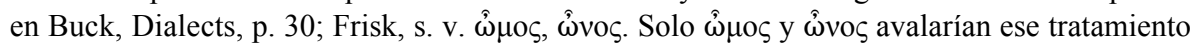
y para $\hat{\omega} \mu$ o parece imposible determinar si hay que partir de una forma básica con vocalismo largo *omsos. Para la doble posibilidad oNs $>$ oN, > oNN cf. Darms Vrddhi p. 325.

EMERITA. Revista de Lingüística y Filología Clásica (EM) - LXV 2, 1997, pp. 221-256 
M. P. Hualde Pascual - Eolismos en Jonia: Revisión de un problema de geografía intradialectal

/oNs/ > /O:N/, que daría cuenta de las formas del jónico-ático; (2) /oNs/ > /oNN/ que daría cuenta de las formas lesbias. (b) Partir de diferente cantidad vocálica para las formas del lesbio y del jónico-ático: un grado breve *,!snapara el lesbio ovva y un grado largo *,ōsna- para las formas del tipo §vos, presentes en jónico-ático ${ }^{50}$.

Una reciente explicación de Ringe ${ }^{51}$, que trata de dar cuenta de ambas formas, del jónico-ático y del lesbio, supone que el verbo §véoual sería originariamente un verbo denominativo sobre un término base *ovos (de *,!sno-, con grado breve original de la raíz): óvéouar (de *,!sne+e/o- ${ }^{52}$ ) y sólo por analogía del grado largo de su antónimo $\pi \omega \lambda \varepsilon \dot{\omega} \omega$ surgiría el grado

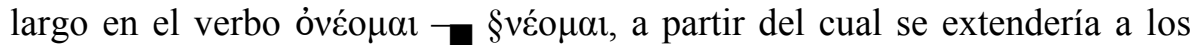
sustantivos $\hat{\omega} v o \varsigma, \S v \eta ́$ de forma secundaria. El lesbio no participaría de este cambio analógico debido a que la forma esperable en este dialecto *őn $\eta \mu \alpha 1$ por su diferencia formal con el antónimo * $\pi \omega ́ \lambda \eta \mu \mathrm{l}$ (frente al jon.át. ỏvદ́o $\mu \alpha l$ $\pi \omega \lambda \varepsilon \dot{\varepsilon} \omega)$ dificultaría dicha influencia analógica ${ }^{53}$.

50 Esta explicación remonta a F. Solmsen, «Sigma in Verbindung mit Nasalen und Liquiden im Griechischen», KZ 29, 1888, pp. 59-124, 329-58; M. Lejeune, Phonétique historique du Mycénien et du Grec ancien, Paris, 1972, p. 123; Chantraine, reseña a Buck, The Greek Dialects, en $R P h$ 31, 1957, pp. 98-100.

51 D. A. Ringe, en su tesis The perfect tenses in Greek inscriptions, Diss. Yale, 1984, pp. 81-85 y art. cit., pp. 45-56.

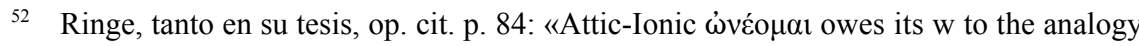
of its antonym $\pi \omega \lambda \varepsilon \dot{\varepsilon} \omega$ 'sell', and this $\omega$ was then introduced into the noums $\omega v \eta$ 'sale' and wvos 'price' from the verb [... . ] and the pre-form will have been *wosn-, with short *o», como en su artículo art. cit. p. 52: "Originally there was a basic noum ōvos 'price' $\left(<60<62>{ }^{*}\right.$ wosnos) with a deverbative *ōv 'sale', 'contract' (*wosna) and a denominative verb *ōveo $\mu a \mathrm{r}$ 'buy' (*wosneye/o). In the verb initial *O was replaced by $\omega$ under the influen-

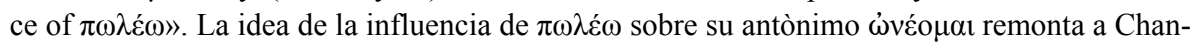
traine, «A propos de grec SNEOMAI», Scritti in onore di Giuliano Bonfante, Vol I, Brescia

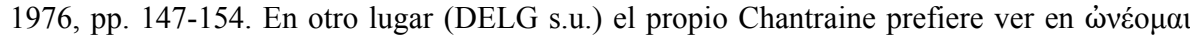
un deverbativo iterativo con grado largo -o- de la raíz como $\pi \omega \lambda \varepsilon \dot{\varepsilon} \omega$. Según Ringe, sobre un deverbativo iterativo contracto se esperaría un sustantivo con base - $\eta$ - como $̋ v \eta \sigma ı \varsigma, ~ ڤ ँ v \eta \mu \alpha$, términos que aparecen asimismo documentados. Por el contrario cree que $\hat{\omega} v o \varsigma$ es el término básico por comparación con otras lenguas IE.

53 Sentada esta base acerca de las dificultades etimológicas del término, siempre según

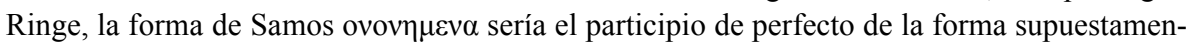

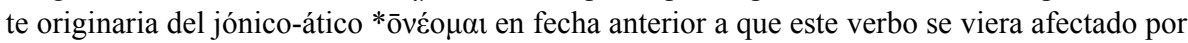

EMERITA. Revista de Lingüística y Filología Clásica (EM) - LXV 2, 1997, pp. 221-256 
M. P. Hualde Pascual - Eolismos en Jonia: Revisión de un problema de geografía intradialectal

En cualquier caso, dada la forma epigráfica ONONHMENA y dado el carácter arcaico de la inscripción en que aparece (580-560 en la datación de la ed. pr. frente a la datación de Jeffery que la sitúa ca. 525) ${ }^{54} \mathrm{y}$, puesto que esta época aún no se ha regularizado en Jonio de Samos la utilización de dígrafo $<$ ov $>$ para la notación de la larga secundaria ${ }^{55}$, cabría entender que ambas $<_{0}>$ de ovov $\eta \mu \varepsilon v \alpha$ representan dos vocales breves, si bien, en este caso, tendríamos la dificultad de tener que explicar un tipo de reduplicación, inusual en el perfecto en griego, consistente en la simple repetición de la pri-

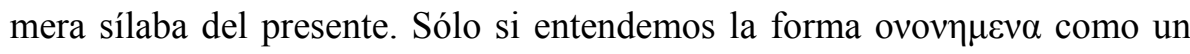
caso de reduplicación a partir de la primera sílaba del presente, se puede de-

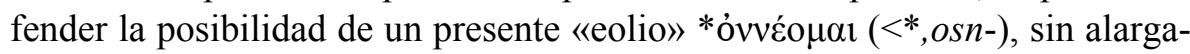
miento compensatorio y la consiguiente geminación: ovov $(v) \eta \mu \varepsilon v \alpha$ (frente al

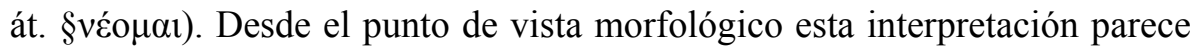
difícil de sostener por el aislamiento de este tipo de reduplicación en griego, pero, sobre todo, porque, en última instancia, la grafía no permite constatar la mencionada reduplicación. Parece, pues, que no contamos con argumentos suficientes para defender la forma ovovๆ $\mu \varepsilon v \alpha$ de Samos como uno más de los supuestos eolismos de Jonia, pero aún cabría señalar que, en el caso de que se aceptara para la forma de Samos un tratamiento fonético lesbio del grupo /ons/ > /onn/, ovov(v) $\eta \mu \varepsilon v \alpha$, se trataría de un eolismo presente en la variedad dialectal del jonio de Perinto, no compartida por el jonio de Ampurias, en el cual se documenta la resolución del grupo /ons/ con alargamiento compensatorio /O:n/ en el infinitivo de perfecto de este mismo verbo

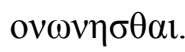

2.2. Contexto de segundo alargamiento compensatorio resuelto por diptongación.

la extensión analógica del vocalismo largo.

54 Datación de Jeffery, LSAG 371, n. 35, con argumentos que no convencen a A. J. Graham, art. cit., p. 74, n. 17.

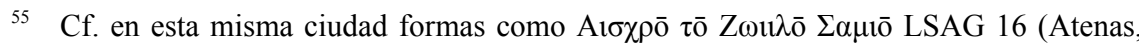
490-480); aún en el siglo IV: $\tau \bar{̄} \varsigma$... $\alpha \gamma \rho \bar{c} \varsigma$ IPr. 369. 16/17 (Tebas junto a Micale, ca. 350),

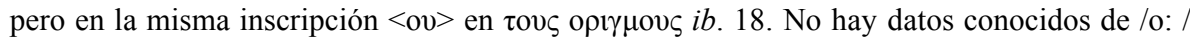
procedente de primer alargamiento por compensación en época arcaica entre las inscripciones de Samos.

EMERITA. Revista de Lingüística y Filología Clásica (EM) - LXV 2, 1997, pp. 221-256 
M. P. Hualde Pascual - Eolismos en Jonia: Revisión de un problema de geografía intradialectal

El segundo alargamiento compensatorio está generalizado en las ciudades de la Dodecápolis, tanto en interior de palabra como en posición final desde los más antiguos testimonios: cf. en posición interior

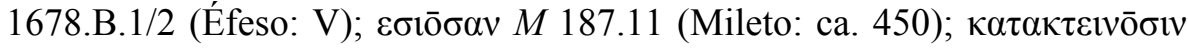

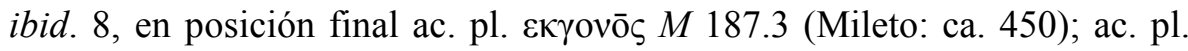
$\mu v \varepsilon X_{\varsigma} I E p h .1 . B .2 .4$ (Efeso: VI); part. $\tau \rho \alpha \varphi Z_{\varsigma} I E K$ 2.B.21/2 (Eritras: a. 454) et al.

Sin embargo, hay cinco ejemplos de diptongación para la resolución del grupo - $n s$ - interior, siempre en la desinencia de tercera persona de plural: en Quíos se documenta, en una sóla inscripción, en el siglo $\mathrm{V}$, la diptongación en dos ocasiones, $\pi \rho \eta \xi$ oı ib. B.15/16. Asimismo se documenta en Mileto, según la lectura de Herrmann, el subjuntivo $\theta \varepsilon \omega 1 \sigma \mathrm{r}$ Klio 52, 1970, p. 166, 1.34, (Mileto: V 2a m.) ${ }^{56}$. En los últimos años se ha dado a conocer una forma procedente de Ampurias (colonización focense), que muestra una diptongación semejante a las tan conocidas formas de Quíos, en la tercera persona del subjuntivo del

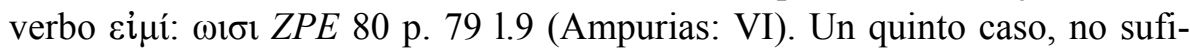
cientemente resaltado ${ }^{57}$, se documenta en Tasos $\lambda \alpha[\gamma] \chi \alpha v$ or $\sigma$ s Sokolowski LSCG 113 (Tasos: ca.475). Esta forma, inicialmente interpretada como error del lapicida ${ }^{58}$, y posteriormente como optativo ${ }^{59}$, ha sido revisada e interpretada, con argumentos convincentes ${ }^{60}$, como indicativo, con resolución dip

56 Cf. P. Herrmann, «Zu den Beziehungen zwischen Athen und Milet im 5. Jahrhundert», Klio 52, 1970, pp. 163-173. Asimismo Herrmann propone restituir otra forma verbal con desinencia lesbia en $\alpha v \alpha \gamma \rho \alpha[\psi \omega \imath \sigma ı v]$ ib. 1. 3 .

57 Sí lo recoge A. Hernández Vázquez, art. cit., p. 288 y n. 37.

58 Cf. C. Rolley, «Le sanctuaire des dieux patrôoi et le Thesmophorion de Thasos», BCH 89, 1965, pp. 441-483.

59 Cf. M. Guarducci Epigrafia Greca IV p. 12, quien lee el texto como A@HNAIHI

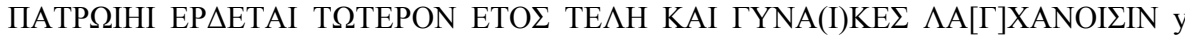
traduce «ad Atena Patroia si sacrifica un anno sì e un anno no. Anche donne ottengano (porzioni dei sacrifici). »

60 R. Arena, «Zu einer thasischen Inschrift» ZPE 49, 1982, p. 140, argumenta que la interpretación de Guarducci de $\lambda \alpha \gamma \chi \alpha$ oı $\sigma$ vv como optativo estaría fuera de lugar, puesto que lo esperable sería la forma $\lambda \alpha \gamma \chi \alpha ́ v o \iota \varepsilon v$; además, la interpretación de esta forma como indicativo se adecua más a la primera forma verbal que aparece en el texto en indicativo $\varepsilon \rho \delta \varepsilon \tau \alpha$. Finalmente, la presencia constatada de formas en -oıol para la $3^{\mathrm{a}}$ pl. en jonio hace verosímil di-

EMERITA. Revista de Lingüística y Filología Clásica (EM) - LXV 2, 1997, pp. 221-256 
M. P. Hualde Pascual - Eolismos en Jonia: Revisión de un problema de geografía intradialectal

tongal del grupo -ns-, como posible eolismo en la isla de Tasos ${ }^{61}$.

Es, no obstante, curiosa la convivencia de formas con alargamiento y formas con diptongación en un mismo texto, como en el caso de la mencionada inscripción de Quíos en las formas $\tau \circ \varsigma$ B.5, C.1; $\tau \mathrm{X}_{\varsigma} \chi 0 \rho \mathrm{X}_{\varsigma} \mathrm{B} 7 / 8$ y para el grupo - $n s$ - interior en $\pi \mathrm{X} \sigma \alpha$ A.9, junto a la diptongación en $\lambda \alpha \beta \omega 1 \sigma \mathrm{l}$ B.15.16, $\pi \rho \eta \xi o t \sigma ı v ~ A .17 / 18.20$. Este hecho, lejos de resultar una incoherencia, avala la idea de eolismo en Jonia: las formas en -oıø, que en lesbio son el resultado de un proceso fonético (la diptongación), en jonio parecen ser formas mixtas, resultado de la adaptación del final -oırl a formas jonias, a favor de lo cual hablaría la / $/$ :/ procedente de /a:/ y la $v$ eufónica de la forma $\pi \rho \eta \xi o l \sigma v^{62}$. Nos encontraríamos, pues, ante un lesbismo en jonio oriental que se trata, en concreto, de un préstamo morfológico, no fonético, puesto que el segundo alargamiento por compensación se cumple de forma habitual y únicamente en el contexto morfologico de tercera persona de plural se adopta la forma lesbia -oıøl, - $\omega \iota r \mathrm{l}$ en las ciudades de Quíos, Focea, Mileto y Tasos. Lamentablemente, no tenemos más datos arcaicos de tercera persona de plural en Quíos, Focea y Tasos que nos permitan comprobar el asentamiento de esta forma desinencial en la variedad dialectal de dichas ciudades. Sin embargo, no deja de ser interesante el hecho de que sí aparezca la desinencia de $3^{\mathrm{a}} \mathrm{pl}$. en su forma jonia esperable -ovol en inscripciones arcaicas

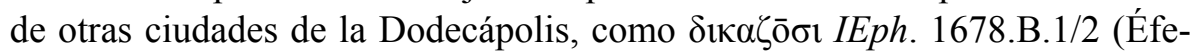
so: V) y, sobre todo, llama la atención la fluctuación entre la forma lesbia y la forma jonia en las inscripciones de una misma ciudad, Mileto, y en una

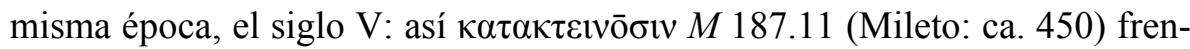
te a $\theta \varepsilon \omega 1 \sigma \mathrm{r}$ Klio 52,1971 , p. 163 , (Mileto: V 2a m.). Esto nos lleva a pensar que las formas con diptongación son préstamos puntuales que no llegaron a integrarse plenamente en el sistema del dialecto jonio de Asia.

\subsection{Presencia de /a:/ en época arcaica.}

cha interpretación.

${ }^{61}$ No son desconocidos otros elementos comunes entre el jonio de Tasos y el del norte de la Dodecápolis, así las formas en ịó (cf. Hernández Vázquez, art. cit., p. 288) o el genitivo en -eu para los masculinos de temas en -a, cf. $\Lambda \varepsilon \omega v 1 \delta \varepsilon v$ SEG XXXI. 782 (Tasos) junto a

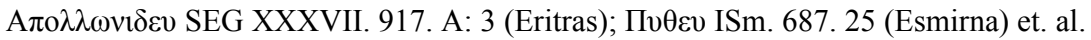

62 Cf. Hernández Vázquez, art. cit., p. 289.

EMERITA. Revista de Lingüística y Filología Clásica (EM) - LXV 2, 1997, pp. 221-256 
M. P. Hualde Pascual - Eolismos en Jonia: Revisión de un problema de geografía intradialectal

El jonio de la Dodecápolis, al igual que el jonio insular, presenta /ع:/ procedente de /a:/, incluso detras de /e/, /i/ o /r/, frente al proceso de Rückverwandlung experimentado por el ático. Las formas en /ع:/ procedente de /a:/ son constantes en las inscripciones jonias hasta en siglo IV, fecha en la que, con el desarrollo y expansión de la koiné, la mayor parte de las ciudades de la Dodecápolis empiezan a documentar formas con /a:/ tras /e/, /i/ y /r/. Muy distinto es el caso sorprendente de la presencia de /a:/ en esta posición, en algunas ocasiones, en época arcaica (s.VI-V), fecha en la que no parece fácil el recurso a la influencia de la koiné para su explicación y que podrían ser un hecho de arcaísmo compartido con el vecino dialecto lesbio. Los datos manejados en este sentido pertenecen a dos cronologías diferentes, que abordaremos por separado: una más arcaica (s. VI-V) y otra posterior (V-IV in.):

(a) Los datos más antiguos (s. VI-V med.) aparecen en Samos, Quíos, Antípolis y Ampurias (estas dos últimas de colonización focense): En Antípolis se documenta para el genitivo del nombre 'diosa' una forma gen. $\theta \varepsilon \alpha \varsigma L S A G$ p. 288 n.3 (Antípolis: 450-425); igualmente con /a:/ aparece la forma de teónimo en Quíos dat. A yvaıaı SEG XVII 379.5 (Quíos, V p.pr.); sorprendente es, asimismo, la forma

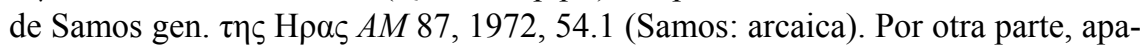

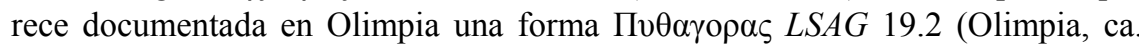
470), para el nombre del cantero samio que realizó la inscripción. La forma de Antípolis $\theta \varepsilon a \varsigma$, resulta llamativa, ya no sólo por su timbre, sino por su formación, ya que para el nombre de la diosa se esperaría en jonio $\dot{\eta} \theta \varepsilon o ́ \varsigma$ y no es improbable que este término presente influencia de la lengua homérica. Para ПvӨ $\alpha \gamma o \rho \alpha \varsigma$, nombre del lapicida samio en Olimpia, no puede descartarse que haya recibido la influencia del dialecto occidental de la ciudad en que se escribió, pese a tratarse de un antropónimo. La forma Hpas, documentada en una breve inscripción dedicatoria recogida por Dunst ${ }^{63}$ entre una relación de inscripciones arcaicas, aparece sobre un pequeño caldero de bronce de tipo samio conservado en el museo de Berlín, pero carece de procedencia concreta y datación precisa. Ello permite suponer que tenga un origen foráneo, $\mathrm{o}$, en cualquier caso, que sea un ejemplo de aticismo temprano en las inscripciones de Samos. El teónimo de Quíos dat. A pooral SEG XVII 379.5 ha sido también interpretado como aticis$\mathrm{mo}^{64}$, aunque, si nos atenemos a la datación que Forrest hace de la inscripción, situándola en fecha algo anterior al siglo $\mathrm{V}$, resulta difícil mantener el recurso a

63 G. Dunst, «Archaische Inschriften aus Samos», AM 87, 1972, pp. 99-163.

${ }^{64}$ K. Stüber, op. cit., p. 23.

EMERITA. Revista de Lingüística y Filología Clásica (EM) - LXV 2, 1997, pp. 221-256 
M. P. Hualde Pascual - Eolismos en Jonia: Revisión de un problema de geografía intradialectal

la influencia externa, a lo que se añade la dificultad de que el prestigio del ático haya impuesto su vocalismo al nombre de divinidades de arraigada tradición local.

La única confirmación sin duda de la presencia de /a:/ sin cierre en /ع:/ apare-

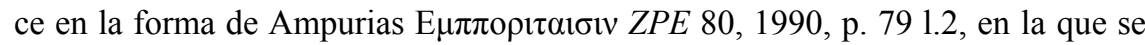
documenta la desinencia de dat. pl. - $\alpha \_\sigma v$, sin duda eolia ${ }^{65}$, frente a la jonia esperable $-\eta \iota \sigma v^{66}$. De nuevo, como en el caso de la resolución por diptongación del contexto de segundo alargamiento compensatorio, nos encontramos con un hecho fonético ligado al préstamo de una marca morfológica. En este caso, no tenemos más datos de dat. pl. de temas en -a en inscripciones arcaicas de origen focense,

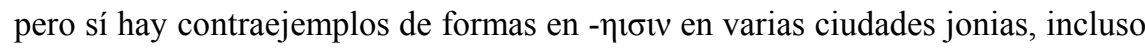
en aquellas que presentan otros casos de lesbismo en sus inscripciones, como el caso de $\eta \mu \varepsilon \rho \eta[1] \sigma w v ~ D G E 688$ B 4/5 (Quíos: V).

(b) Junto a estos casos confirmados de /a:/ conservada, aparece otro bloque de datos, documentado en antropónimos, en fecha algo más reciente (s. V/IV), en la ciudad de Eritras. Estos nombres, que presentan una /a:/, en la mayor parte de los casos tras /e/, /i/, o /r/, han sido considerados ${ }^{67}$ como eolismos residuales en Eritras, si bien la observación del texto de la inscripción así como la atención a la fecha que le atribuyen los editores hace más que probable que se trate de simples formas de influencia ática o producto de la temprana imposición de la koiné en la ciudad.

Las más antiguas formas con /a:/ en Eritras son de finales del siglo V o comienzos del IV, en dos inscripciones, IEK 1 y IEK 152: En la primera de ellas se documenta $\mathrm{A} \pi \varepsilon \lambda \lambda \lambda_{1}$ ऽ IEK 1.1 (V-IV), si bien en el texto aparecen otras formas con /e:/, como $\mu \eta \delta \varepsilon \mu \eta \eta$ ib. 4, $\tau \alpha \mu \eta \eta$ ib. 7. En la segunda inscripción mencionada

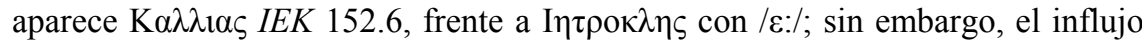

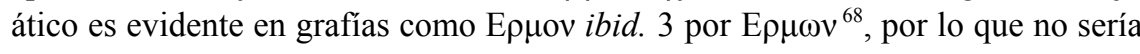
raro que también al ático se debiera la /a:/ de los mencionados antropónimos.

Bastante posterior es una inscripción, IEK 161, datable en la primera mitad

${ }^{65}$ Cf. E. Sanmartí - R. A. Santiago, «Une lettre grecque sur plomb trouvée à Emporion (Fouilles 1985)» ZPE 68, 1987, p. 121.

66 Documentada en otras ciudades de la Dodecápolis, incluso en las ciudades más cerca-

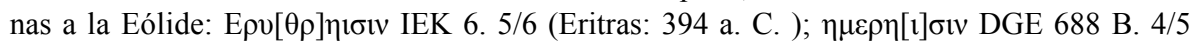

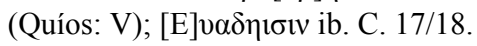

${ }^{67}$ Garbrah, o. cit., p. 15 y 84.

68 Cf. el comentario de Engelmann y Merkelbach a la inscripción IEK 152.

EMERITA. Revista de Lingüística y Filología Clásica (EM) - LXV 2, 1997, pp. 221-256 
M. P. Hualde Pascual - Eolismos en Jonia: Revisión de un problema de geografía intradialectal

del siglo IV, en la que se documentan asimismo $[\Lambda \alpha] \mu \beta \rho \alpha \gamma o \rho \alpha \varsigma$ (13) y [T] $\operatorname{cop} \rho \alpha \varsigma$ (20), sin que en el texto aparezcan otras formas de koiné. A las mencionadas formas con /a:/ se añaden las formas que aparecen en inscripciones nu-

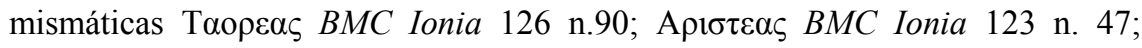

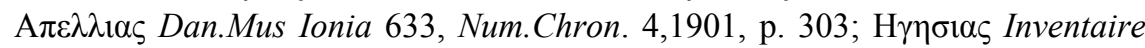
Waddington 1658, que pueden recibir la misma explicación, como elementos de koiné.

En cualquier caso, no hay que dejar de observar que los antropónimos son un tipo de palabras que tienen la peculiaridad tanto de reflejar en fecha temprana las innovaciones, como de conservar por mucho más tiempo el arcaísmo, y de esta manera, también en otras ciudades jonias aparecen desde finales del siglo $\mathrm{V}$ y

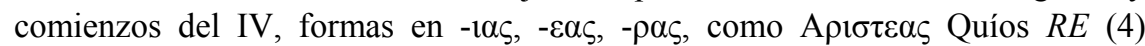
(V/IV), Delos ID 104-21 b B 10 (340 a.C.), Bı [s] DGE 688 c.25/26 (Quíos: V);

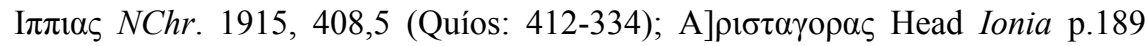
(Mileto: ca. 350) et al.

Más posibilidades de interpretación como eolismo, puesto que no son atribuibles al ático o a la koiné, tendrían el genitivo Пv $\theta \varepsilon \alpha$ IEK 22.73 (Eritras: IV ex.) y BMC Ionia 126 n. 85, forma indudablemente no jonia, o el antropónimo Izpoitas IEK 201.C.54.56 (Eritras: 300- 260). No obstante, al tratarse de antropónimos siempre cabe la posibilidad de que designen a un individuo de origen extranjero, sea su origen eolio o no lo sea o que, como dijimos supra, respondan a un hecho de moda ${ }^{69}$. En cualquier caso, cabe señalar que otras ciudades de la

69 Garbrah, o. cit., p. 15 n. 4, considera fuera de cuestión la posibilidad de que estas formas se deban a otro dialecto distinto que el eolio (rechaza explícitamente la posibilidad de dorismo). Ante esto cabe argumentar que en jonio de Asia aparecen formas de antropónimo que probablemente no son jonias pero tampoco pueden deberse a préstamo o sustrato eolios:

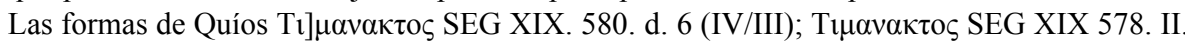

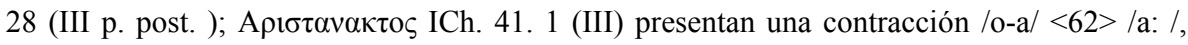
ajena al jonio (cf. las formas habituales E $\mu \omega v \alpha \xi$ SEG XIX. 580. C. II. 7 (Quíos: IV/III) et. al. ). Esta contracción no se produce en lesbio, por lo que es preciso descartar la condición de eolismo de estas formas; por el contrario la contracción /o-a/ $<62>$ /a: / se produce en los dialectos occidentales. Ciertamente nada en las inscripciones en que aparecen dichos antropónimos hace suponer que designen a individuos extranjeros, así que no se podría descartar la presencia de un elemento occidental en la antroponimia de Quíos, siempre que no se trate de formas con elisión de la última vocal del primer término del compuesto. No obstante, al movernos en el terreno de los antropónimos cualquier afirmación al respecto debe ser cuidadosamente matizada. Sobre el elemento occidental en la Dodecápolis, Sakellariou, o. cit., p. 290 n. 2.

EMERITA. Revista de Lingüística y Filología Clásica (EM) - LXV 2, 1997, pp. 221-256 
M. P. Hualde Pascual - Eolismos en Jonia: Revisión de un problema de geografía intradialectal

Dodecápolis presentan también formas similares, así $\Phi$ \} \lambda \omega \tau \alpha \varsigma \text { SEG XIX } 580.C.II.5 (Quíos: IV/III) o el genitivo K $\tau$ бıа M 122.II.26 (Mileto: 369/8), sin que tenga justificación la atribución de un origen eolio a estas formas para su explicación ${ }^{70}$.

(c) A estos datos se añade el topónimo de Eritras Пo $\lambda \imath \chi v \alpha$, Athenian Tribute Lists, formado sobre el diminutivo $\pi \mathrm{o} \lambda \dot{i} \chi v \alpha$, con sufijo *-sna, 'ciudadela', que, en apariencia, conserva una /a:/, si bien distintos textos literarios nos han transmiti-

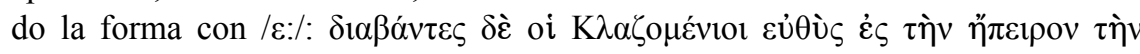

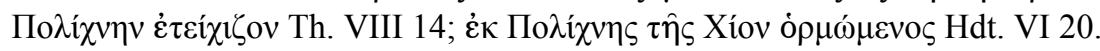

\subsection{Contracción /a:-o/> /a:/.}

Se ha defendido una supuesta contracción ${ }^{71} / \mathrm{a}:-\mathrm{o} />$ /a:/, de origen eolio, en los antropónimos de Eritras $\Lambda \alpha \mu \varepsilon \delta \omega v$, Dan.Mus Ionia 601 y ZN 14, 1886, 151 y $\Lambda \alpha \pi \rho \varepsilon \pi \eta \varsigma$ Dan.Mus. Ionia 685 , y en el apelativo ac. pl. $\lambda \alpha \tau v \pi \mathrm{ov} \varsigma$ IEK 22.5.32.41.74 (Eritras: IV). Respecto a la posibilidad de eolismo en las dos formas de antropónimo, repetimos la idea expuesta en el punto anterior: cualquier forma de antropónimo debe considerarse con extrema prudencia por la posibilidad de que designe a un individuo no jonio, y en especial las formas que nos ocupan, documentadas sobre moneda, por el carácter viajero de las mismas. Tampoco es absolutamente descartable la posibilidad de que nos encontremos ante formas haplográficas, con eliminación de la última vocal del primer elemento, a partir de formas, frecuentes en Jonia, con primer elemento $\Lambda \alpha o-$ esclerotizado y sin cumplimiento de la metátesis de cantidad, si bien esta exlicación es más complicada.

Es, no obstante, en la forma $\lambda \alpha \tau v \pi 0 u \varsigma$ 'talladores de piedra', donde la posibilidad de eolismo está, a mi entender, totalmente descartada: el término consta de un primer elemento creado sobre $\lambda \hat{\alpha} \alpha \varsigma$, ac. $\lambda \hat{\alpha} \alpha v$ 'piedra', término de etimología incierta ${ }^{72}$ y que sólo secundariamente conoce una variante te

\footnotetext{
70 Para este tipo de formas de genitivo en - $\alpha$ en el dominio jonio cf. C. Brixhe - A. Panayotou, «Atticisation de la Macédoine», Verbum 11, 1988, p. 251.

71 Garbrah o. cit. pp. 15, 43, 145, López Eire, art. cit. p. 165.

72 Cf. Chantraine DELG s. v. $\lambda \hat{\alpha} \alpha \varsigma$, sobre la oscuridad de la estructura y de la declinación de este término, quien recoge las interpretaciones antiguas de Brugmann $I F$ 11, 1900, p. 100 ss. y Benveniste Origines 33, que operan ambas con -,- intervocálica y que son descartdas a la vista del chipr. $\Lambda \alpha \mathrm{o}(\varsigma)$ ICS 84 y mic. ra-e-ja, sin -,-. Asimismo reproduce la opinión 
M. P. Hualde Pascual - Eolismos en Jonia: Revisión de un problema de geografía intradialectal

matizada $\lambda \hat{\alpha} \mathrm{o} \varsigma$ ac. $\lambda \hat{\alpha} o v^{73}$. No obstante, las formas de compuesto con el primer elemento $\lambda \alpha$ - esperable están generalizadas tanto en textos epigráficos como literarios: en concreto, el término que nos ocupa $\lambda \alpha$ ótv $\pi$ o $\zeta$ aparece en prosa jonia, Hp., Fract. 31, y, fuera de ella, entre otros, en Sófocles, fr. 530, frente a la forma con primer término tematizado $\lambda \alpha$ ó $\tau$ to $\delta$, presente en la Antología Palatina VII 429.3 y 4.59. Es preciso decir, además, que este mismo primer elemento de compuesto se encuentra dentro de la Dodecápolis en la forma de Éfeso ac. pl. $\lambda \alpha \tau o \mu 1 \alpha$ IEph. 3.11 (Éfeso: III) 'canteras de piedra', sin que nada haga pensar en la posibilidad de eolismo para ninguna de ambas formas de la Dodecápolis.

\subsection{Palatalización de la dental sonora /d/ en contacto con yod secundaria.}

La palatalización de la dental sonora /d/ en contacto con yod secundaria, documentada en lesbio literario y epigráfico, es uno de los hechos que se han pretendido ver como eolismo en jonio de Asia Menor. Un solo dato del jonio de Asia ha respaldado esta posibilidad, la forma fragmentaria documentada sobre una estatera (s. VI?) de Focea Ziovv- SGDI 5623 (Focea: s.d.), pretendidamente para el nombre de Dioniso. En efecto, esta forma, que parece presuponer la palatización de la dental sonora /d/ en contacto con /i/, se ha considerado como eolismo en la Jonia del norte, a la vista del paralelismo con

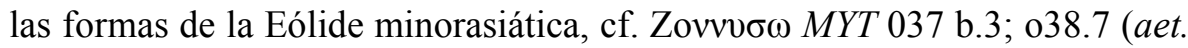
imp.).

Varios son, no obstante, los problemas a la hora de la interpretación de la forma focense como lesbismo:

(a) Por una parte, el proceso fonético de la palatalización de /d/ ante yod secundaria en lesbio ha dado un paso más y la yod se funde por completo con la consonante anterior ${ }^{74}$, según lo cual aparecen formas en lesbio epigráfico como el

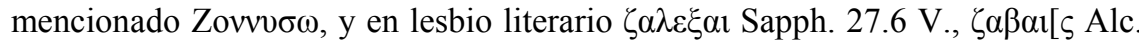

de Heubeck, IF 66,1961, pp. 29-34, quien descarta la presencia de -,- sin pronunciarse sobre la etimología.

73 Así, en un compuesto similar, aparece la forma $\lambda \alpha \xi$ óo $\varsigma$ 'tallado en piedra' en Sófocles, fr. 212, pero en época helenística $\lambda \alpha 0 \xi$ óo $($ pap. III a. C. ).

74 Cf. Hernández Vázquez, art. cit., p. 288.

EMERITA. Revista de Lingüística y Filología Clásica (EM) - LXV 2, 1997, pp. 221-256 
M. P. Hualde Pascual - Eolismos en Jonia: Revisión de un problema de geografía intradialectal

38 a, 3 V.; así, la consonantización de /i/ en hiato, produce, en definitiva, la pérdida de una sílaba, según constata el metro en los autores eolios ${ }^{75}$. Es interesante el hecho de que este fenómeno sólo aparece en fragmentos de los líricos y en algunas inscripciones de la Eólide de época imperial, pero no en inscripciones propiamente dialectales.

(b) Hay que tener en cuenta el aislamiento de la forma fragmentaria Zıovv-, atribuida a la ciudad de Focea. Esta forma se ha transmitido gracias a la lectura de Riggauer, a partir de la publicación del texto por Meister ${ }^{76}$, y ha sido posteriormente recogida por Bechtel en SGDI 5623. No obstante, el desciframiento de Riggauer no permite leer el término completo, sólo el fragmento Zıovv, y otros autores presentan lecturas distintas, así Babelon en su Traité des monnaies II,1 n. 169, de 1907, recoge la lectura Tios frente a Head Zıos ${ }^{77}$. En definitiva, es significativo el posible carácter foráneo que confiere a la forma Ziovo su aparición sobre moneda, a lo que se unen la ausencia de lectura y datación seguras (recuérdese que despues de la edición de Bechtel en Sammlung y de Schwyzer $D G E$ no ha habido revisión ni posterior edición del texto) ${ }^{78}$.

(c) Por último, la ausencia de formas en Zıovvv-, Zovvv- en eolio epigráfico antes del siglo II d.C., hace este dato sospechoso, al que, en cualquier caso, hay que considerar con las debidas precauciones. En este sentido, es interesante recordar las conclusiones de $\operatorname{Hodot}^{79}$ acerca de este proceso de palatalización en las inscripciones del eolio de Asia:

(1) la palatalización que se da en $\delta 1 \alpha>\zeta \alpha$ tiene su origen en la tendencia al monosilabismo que manifiesta esta preposición, la única que presenta un hiato interno.

(2) en el nombre de Dioniso la palatalización está motivada por la etimología incierta del teónimo, que, en cualquier caso, es sentido como extranjero. No

75 Cf. Hodot, o. cit., pp. 42-43 y n. 10, quien resalta la doble posibilidad de utilización de las formas disilábicas y monosilábicas según las necesidades métricas por parte de Alceo y Safo. Las condiciones en las que la tradición de los líricos se inclina por la reducción del grupo /di/ responderían no tanto a una ley fonética como a una «règle d'apellation», a partir de su caracterización léxica (Hodot o. cit. y n. 12).

76 Cf. R. Meister, Philologus 1890, pp. 610-611.

77 La información es tomada de Hodot, o. cit., p. 44 n. 19. Tal vez Babelon desconoce el articulo de Meister y de ahí que transcriba la forma Tios que recoge la lectura de Sestini de 1817.

78 Cf. las observaciones de Hodot en López Eire, art. cit., p. 178.

79 Cf. Hodot, o. cit., pp. 44-45.

EMERITA. Revista de Lingüística y Filología Clásica (EM) - LXV 2, 1997, pp. 221-256 
M. P. Hualde Pascual - Eolismos en Jonia: Revisión de un problema de geografía intradialectal

obstante, formas en $\Delta 10^{\circ}, \Delta 10 \sigma^{\circ}$ se documentan desde los primeros testimonios epigráficos tanto en la Eólide como en la Dodecápolis.

(3) el fenómeno de la palatalización de /di/ se observa esporádicamente en otros dialectos, en los que la posibilidad de eolismo queda fuera de lugar:

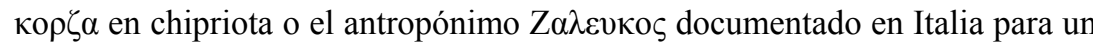
individuo locrio (Bechtel $H P N$ p. 184).

Ateniéndonos, pues, al propio carácter del proceso de palatalización de /di/ en eolio, recientemente estudiado por Hodot, y a lo débil del testimonio de la inscripción monetaria de Focea, parece difícil asegurar la posibilidad de eolismo en jonio para la forma Zovo.

\subsection{Reducción del grupo -sthl- $a$-sl-.}

Es sabido que el grupo *-sthl-, presente en el adjetivo $\dot{\varepsilon} \sigma \theta \lambda$ ó $^{\circ}$ 'excelente, noble', aparece simplificado como $-s l-\dot{\varepsilon} \sigma \lambda{ }^{\circ} \varsigma$, en varios dialectos, entre ellos en jonio de la Dodecápolis, hecho interpretado asimismo como eolismo en Jonia $^{80}$, dada la presencia de formas en $\varepsilon \sigma \lambda o-$ en los poetas lesbios. Si bien es cierto que dicho adjetivo no aparece con frecuencia en las inscripciones griegas, tenemos, sin embargo, la preciosa documentación que nos aportan los antropónimos creados sobre él. Hasta hace pocos años, no obstante, los testimonios epigráficos de antropónimos en $\dot{\varepsilon} \sigma \lambda 0^{\circ}$ eran escasos, situación que se ha remediado en buena manera con la publicación de varios nuevos en los últimos años ${ }^{81}$, entre los que destacan por su abundancia los documentados en Chipre.

Según el estudio de Masson ${ }^{82}$, frente a las formas en $\dot{\varepsilon} \sigma \theta \lambda \mathrm{o}^{\circ}$, documenta

80 Las formas en $\varepsilon \sigma \lambda 0^{\circ}$ se consideran eolismos en Wilamowitz, Nordionische Steine, Berlín, 1909, p. 10.1; Thumb-Scherer, Handbuch, pp. 245,248; Garbrah, o. cit., pp. 15, 68; López Eire, art. cit. p. 164; Hernández Vázquez, art. cit., p. 288; Stüber, art. cit. p. 82. Por el contrario, Bechtel, Dialekte III, no cita las formas en $\varepsilon \sigma \lambda 0^{\circ}$ entre los posibles eolismos.

81 Cf. O. Masson «Notes d'anthroponymie grecque et asianique 4. Les noms en E $\sigma \theta \lambda$ (o) et $\mathrm{E} \sigma \lambda(\mathrm{o})$ dans les dialectes grecs», $B z N$ 13, 1962, pp. 75-80 y «Anthroponymie, dialectes et histoire», Verbum 10, 1987, pp. 255-256, quien proporciona una panorámica muy detallada del hecho.

82 Esp. Masson, art. cit. en nota anterior y también A. Thompson, «Personal names from ancient Cyprus with ellement E $\lambda \lambda(\mathrm{o})$ Studies in Greek Linguistics Prceed. 8th Annual Meeting Dept. of Linguistics Thessaloniki (April 1987) = Festschrift for J. Chadwick, Salonique,

EMERITA. Revista de Lingüística y Filología Clásica (EM) - LXV 2, 1997, pp. 221-256 
M. P. Hualde Pascual - Eolismos en Jonia: Revisión de un problema de geografía intradialectal

das fundamentalmente en el dominio dorio, en concreto, dentro de la antroponimia de Cirene, Corinto y Creta, aparecen diversos casos de formas en $\dot{\varepsilon} \sigma \lambda 0^{\circ}$, que se documentarían en tres grandes regiones del mundo griego:

(a) en chipriota, numerosos casos de formas en $\dot{\varepsilon} \sigma \lambda 0^{\circ}$ como primer elemento. Este tratamiento sería propio del grupo dialectal arcado-chipriota, si se considera dialectal la forma ${ }^{83}$, documentada en un epigrama hallado en Olimpia, originario de Mantinea de Arcadia, $\eta \varepsilon \sigma \lambda \circ \varsigma$ (Hansen Carmina 380 = IvOl 266);

(b) dentro del jonio, en Eritras, Quíos y Amorgos;

(c) en formas literarias de Safo y Alceo, que no han encontrado correlato en lesbio epigráfico, y, como eolismo, en Pindaro ${ }^{84}$.

Ciñéndonos a los datos concretos del jonio de la Dodecápolis, estos se reducen a dos casos: en Eritras, el antropónimo derivado gen. E $\sigma \lambda \omega v \mathrm{o}[\varsigma$ IEK 327 (Eritras: IV $1 \mathrm{~m}$.); en Quíos, en el adjetivo femenino gen. $\varepsilon \sigma \lambda \eta[\varsigma] D G E$ 689.1 (Quíos: V), documentado en un epigrama sepulcral. Se recordará que ambas ciudades pertenecen a la zona tradicionalmente considerada como de influencia eolia. Dentro del ámbito jonio, pero fuera de la Dodecápolis, se documenta una nueva forma reducida $\dot{\varepsilon} \sigma \lambda o$, en Amorgos $\varepsilon \sigma \lambda \circ \varsigma$ IG XII,7.107 (Amorgos: V) ${ }^{85}$, en jonio de las islas, zona, en principio, ajena a la influencia eolia.

Ciertamente, aunque el término aparece con frecuencia en epigramas, las formas reducidas en $\varepsilon \sigma \lambda \mathrm{o}^{\circ}$ no aparecen motivadas por razones métricas, así

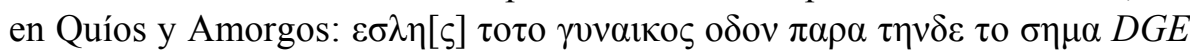

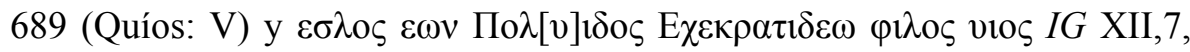
107.1 (Amorgos: V). Sin embargo, sí cabe la posibilidad de que en estos textos la forma en $\varepsilon \sigma \lambda o^{\circ}$ aparezca por influencia literaria, pero este argumento no es aplicable al antropónimo Eø $\lambda \omega v o \varsigma$ de Eritras.

pp. $123-131$

${ }^{83}$ Incluida en el dossier de formas dialectales por L. Dubois, Recherches sur le dialecte arcadien I: Grammaire, Louvaine-la-Neuve, 1986, p. 48. Asimismo L. H. Jeffery, $L S A G$, Oxford, 1990, p. 211 no ve razón que impida la atribución de un origen arcadio a la inscripción, escrita en el alfabeto propio de Mantinea.

${ }^{84}$ Cf. O. Masson, o. cit. en n. 77, p. 79 y «Anthroponimie, dialectes et histoire», Verbum 10, 1987, p. 256.

85 Cf. Knitl, o. cit., p. 58.

EMERITA. Revista de Lingüística y Filología Clásica (EM) - LXV 2, 1997, pp. 221-256 
M. P. Hualde Pascual - Eolismos en Jonia: Revisión de un problema de geografía intradialectal

Dadas las circunstancias y la expansión de las formas en $\varepsilon \sigma \lambda o^{\circ}$ por distintas áreas dialectales, arcado chipriota, eolia y jonia, no es preciso entender que las formas en $\varepsilon \sigma \lambda \mathrm{o}^{\circ}$ de Eritras y Quíos se producen por influencia de la vecina Eólide, sino que puede tratarse, como parece avalar la forma $\varepsilon \sigma \lambda \circ \varsigma$ de Amorgos, de un hecho propio del jonio oriental, compartido a su vez con el dialecto de Lesbos y con el grupo arcado-chipriota o, en cualquier caso, de un hecho resultado de influencia literaria.

\subsection{Tratamiento dorsal de la labiovelar en formas comо око, окобо et al.:}

Las formas con dorsal en el tema del interrogativo-indefinido oко-, frecuentes en textos literarios, se consideran un hecho específico de la lengua de la Jonia del Norte ${ }^{86}$ coincidente con la del lesbio minorasiático oкобov $A I G$ 01,8,9. Así, marginando los datos literarios, que no consideramos probatorios desde un punto de vista dialectal ${ }^{87}$, hay al menos cuatro datos de formas en око- en las ciudades del norte de Jonia y sus colonias: En Eritras oкo1 $\alpha$ IEK 205.11 (Eritras: 380-360) y en las colonias de Focea, en las inscripciones de Ampurias y Pech-Maho publicadas en los últimos años: окобo ZPE 80 (1990) p.79.12 (Ampurias: V); око CRAI 19881.8 (Pech-Maho: 440-450); posiblemente o]ко Almagro IAIGL 21.6 (Ampurias: ca. 450

86 Para la explicación fonética del resultado / $/$ / de la antigua labiovelar no hay unidad de criterios, cf. las distintas explicaciones de: (1) Schulze, $C G A, 1897,908.5$, recogida por Bechtel, Dialekte, p. 89: disimilación de $* \mathrm{k}^{\mathrm{w}}$ entre dos vocales de timbre /o/; (2) Lejeune, Phonétique p. 45: disimilación de la labiovelar a partir de formas negativas del tema del relativo- indefinido, tipo *ouk ${ }^{\mathrm{w}}$ os, con disimilación tras vocal velar oűкos; (3) recientemente, A. Lillo en

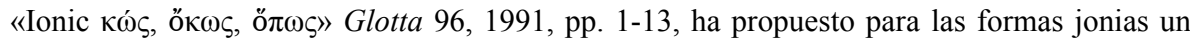
originario ${ }^{*}$ yodk $^{\mathrm{w}} \mathrm{o}$, con asimilación en fase de pervivencia de la labiovelar ${ }^{*}$ yokk $^{\mathrm{w}} \mathrm{o}$. Un complicado juego de reanálisis daría cuenta de las formas en оло- y de la degeminación en las formas en око-.

87 No así Bechtel, Dialekte III, p. 87, quien opera con datos de autores literarios como Arquíloco, Demócrito y Heráclito, en los que observa una reapartición según la cual las formas en gutural aparecen siempre que la labiovelar vaya precedida de vocal posterior /o/, frente a la labial del resto de los casos. En otros autores jonios, sin embargo, prevalecen las formas en gutural, independientemente de si la labiovelar va precedida de /o/ o no (Bechtel op. cit. señala a autores como Herodas, Anacreonte y Heródoto, en los que prevalecen las formas con gutural). En cualquier caso, estas formas literarias no tienen ningún valor probatorio desde el punto de vista dialectal, pues en su aparición pueden intervenir factores como problemas de transmisión de los textos, o, simplemente, el carácter artificial de las lenguas literarias.

EMERITA. Revista de Lingüística y Filología Clásica (EM) - LXV 2, 1997, pp. 221-256 
M. P. Hualde Pascual - Eolismos en Jonia: Revisión de un problema de geografía intradialectal

a.C. ${ }^{88}$. Pese a la coincidencia de las formas del norte de Jonia y de eolio de Egas, tras el estudio de las inscripciones de la Eólide por parte de R. Ho$\operatorname{dot}^{89}$, parece que la forma eolia окоббov es un localismo de Egas, producido por influencia jonia, aislado frente a las más numerosas formas en o $\pi \pi 0-$ del resto de las ciudades eolias. Por tanto, el tratamiento dorsal de la labiovelar en las formas en око- no es un eolismo en jonio, ni un tratamiento común para jonio y eolio, sino, por el contrario, un jonismo en el territorio eolio, o, si se prefiere la formulación, una interferencia fonética del jonio en el dialecto lesbio de Egas.

\subsection{El participio de perfecto flexionado con *-nt-: el caso de Quíos,} $\gamma \varepsilon \gamma \omega v \varepsilon \mathrm{ov} \tau \varepsilon \varsigma$ :

Se suele citar la forma de participio $\gamma \varepsilon \gamma \omega v \varepsilon 0 v \tau \varepsilon \varsigma ~ D G E$ 688.B.12/13 (Quíos: V) como caso de eolismo ${ }^{90}$ dentro del subdialecto de Quíos, opinión esta que se vería respaldada por el hecho de que en esta conocida inscripción, el mojón de demarcación territorial de Lofitis, se concentra la mayor parte de los eolismos habidos en la Dodecápolis jonia. Como es sabido, es una característica de los dialectos eolios la declinación del participio de perfecto como el de presente, con $*_{-} n t$-. No obstante, dificulta notablemente la posibilidad de defender el participio de perfecto en *-nt- como lesbismo en Jonia el hecho de que no se documentan más formas de participio de perfecto en época arcaica y que parece muy improbable que $\gamma \varepsilon \gamma \omega v \varepsilon o v \tau \varepsilon \varsigma$ pueda considerarse como tal. En efecto, la antigua forma de perfecto $\gamma \varepsilon ́ \gamma \omega v \alpha$, de etimología incierta ${ }^{91}$, cuyo pluscuamperfecto $\gamma \varepsilon \gamma \omega \dot{v \varepsilon 1}$ aparece en Homero ${ }^{92}$,

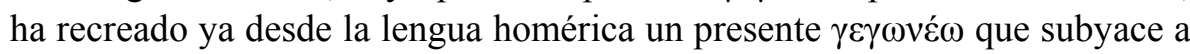

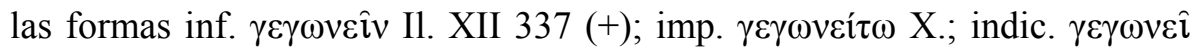

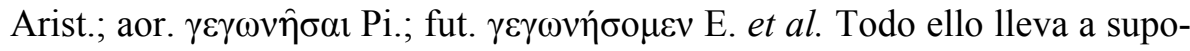

88 Cf. Santiago, art. cit. en n. 4, p. 286 n. 27.

89 Cf. Hodot, op. cit., p. 140.

90 Así López Eire, art. cit., p. 164, compara la forma $\gamma \varepsilon \gamma \omega v \varepsilon o v \tau \varepsilon \varsigma$ con el paso de la flexión de perfecto a presente en eolio como $\pi \varepsilon \varphi \varepsilon \iota \rho \alpha \kappa o v \tau \varepsilon \varsigma D G E$ 596. 4. 5 en Larisa. También Hernández Vázquez, art. cit., p. 289.

91 Cf. Chantraine, DELG s. v. $\gamma \varepsilon \dot{\gamma} \gamma \omega v \alpha$.

92 Cf. Chantraine, Gr. Hom. I, pp. 347-348.

EMERITA. Revista de Lingüística y Filología Clásica (EM) - LXV 2, 1997, pp. 221-256 
M. P. Hualde Pascual - Eolismos en Jonia: Revisión de un problema de geografía intradialectal

ner que el participio $\gamma \varepsilon \gamma \omega v \varepsilon o v \tau \varepsilon \varsigma$ en Quíos sea un caso de participio de presente ${ }^{93}$ con indudable influencia homérica (por lo demás también presente en otros términos de la misma inscripción), hecho que se ve corroborado de manera a mi entender definitiva por la forma de infinitivo presente atestiguada

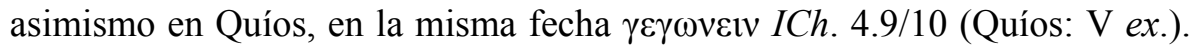
A la vista de los datos, creemos que no hay bases suficientes para defender la existencia de participio de perfecto flexionado como el de presente, con *-nt-, en el subdialecto de Quíos.

\subsection{Conjugación atemática de los uerba uocalia:}

La forma $\delta \psi \alpha \alpha v \tau[1$, documentada en un grafito sobre un fragmento de cerámica en Ampurias, en una breve inscripción de caracteres jonios, ] $v 1$ $\delta ı \psi \alpha v \tau\left[1\right.$ Almagro IAIGL $\mathrm{n}^{\circ}$ 43, es, evidentemente, el dativo de un participio que concertaría con el dativo fragmentario prececente, probablemente un antropónimo, y así su traducción sería «para [-----]on cuando esté sediento», haciendo alusión a la función a la que estaba destinado el vaso cerámico en cuestión. El interés de la forma radica en que al parecer se trata del participio

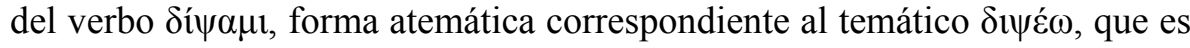
la formación atestiguada en jonio desde Arquíloco y Heródoto. Siendo así que el eolio de Lesbos y de Tesalia presenta en sus dialectos una conjugación atemática para los verbos contractos, esta forma se ha considerado un eolismo en el habla focense de las colonias ${ }^{94}$. Ciertamente el verbo en cuestión plantea algunas reservas, pues a su infinitivo $\delta \iota \eta \hat{v} v$ corresponde un participio en Homero $\delta \imath \psi \alpha ́ \omega v$, posible «eolismo» épico, frente a las formas de $\delta \iota \psi \varepsilon ́ \omega$ mencionadas para el jonio literario. La presencia de una forma atemática en Ampurias parece difícil de justificar, siendo así que el jonio no conoció la extensión de los verbos contractos a los atemáticos, de lo que hay testimonios evidentes desde época arcaica, incluso en las ciudades más septentrionales de la Dodecápolis, aquellas de supuesta influencia lesbia. Así tenemos el caso de part. $\delta \eta \mu \alpha \rho \chi \varepsilon \omega v$ SEG. XVI 485.5 (Quíos: 575/550); part.

93 Así sugerido ya en M. Fuochi, «De titulorum ionicorum dialecto» Stud. ital. di Filol. Class. 2, 1894, p. 252 n. 1 y recientemente D. A. Ringe, The Perfect Tenses in Greek Inscriptions, Diss., Yale, 1984, p. 87.

94 Cf. Santiago, art. cit. en n. 4 p. 290.

EMERITA. Revista de Lingüística y Filología Clásica (EM) - LXV 2, 1997, pp. 221-256 
M. P. Hualde Pascual - Eolismos en Jonia: Revisión de un problema de geografía intradialectal

$\varepsilon \lambda \varepsilon 0 \rho \varepsilon 0 v \tau o \varsigma$ IEK XVII 18/19 (Eritras: V) et al. Una vez más, ante la presencia de numerosos contraejemplos y sin que podamos justificar que se trate de una forma residual en jonio, ni una inscripción foránea, por el carácter inequívocamente jonio de su grafía, debemos suponer que la forma de Ampurias sea un préstamo morfológico puntual en jonio de las colonias focenses.

\subsection{Declinación de los numerales:}

La declinación de numerales se documenta en dos puntos de la Dodecápolis: Quíos y Samos. En Quíos, en la inscripción de Lofitis, que habíamos mencionado como la de mayor concentración de elementos supuestamente eolios, aparecen las formas $\delta \varepsilon \kappa \omega v \delta v \omega v D G E$ 688.D.13/14 (Quíos: V);

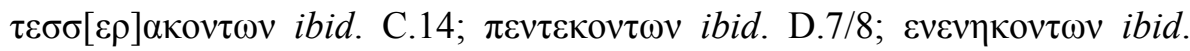
C.24. Por otra parte, en una inscripción hallada en Samos, una dedicación de los Perintios a la Hera samia, aparece la forma paralela $\delta v \omega \delta \varepsilon \kappa \omega v$ SEG XII 391.16/17 (Samos: 580-560). Salvo los datos expuestos, el resto de las formas de numerales que aparecen en los textos epigráficos de la Dodecápolis presentan la forma indeclinable habitual desde los más antiguos testimonios. La flexión de los numerales superiores a cuatro se considera tradicionalmente como un rasgo eolio ${ }^{95}$, no obstante su documentación en este dialecto es

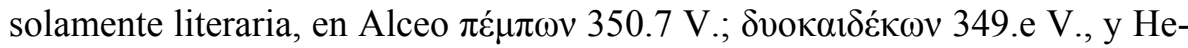

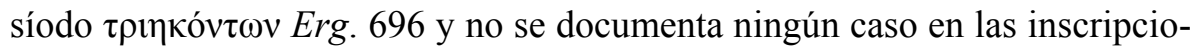
nes de la Eólide ${ }^{96}$. Todo ello lleva a pensar que la hipercaracterización de los numerales con una marca pluralizante es un elemento literario presente en las inscripciones, más que una manifestación de la lengua hablada de la Jonia del Norte. En cualquier caso, es destacable la presencia de este elemento en Samos, de nuevo fuera de la supuesta área de influencia eolia.

\subsection{Adverbio $\alpha \mathrm{l} \varepsilon / \alpha \varepsilon 1$ vs. $\alpha 11$ :}

95 Cf. Roehl, IGA, 1882, p. 107; Buck, Dialects, p. 77; Bechtel, Die Inschriften des ionischen Dialekte, Göttingen, 1887, p. 138; SGDI III, 2, p. 704; Schwyzer, Gr. Gr. I, p. 86; Thumb-Scherer, Handbuch, pp. 359; Sakellariou, o. cit., p. 288; López Eire, art. cit., p. 164; Hernández Vázquez, art. cit., p. 288.

96 Cf. Hodot, op. cit., p. 153, donde da cuenta de la falta de testimonios de esta extensión analógica en las inscripciones del eolio de Asia, así en eolio epigráfico aparece

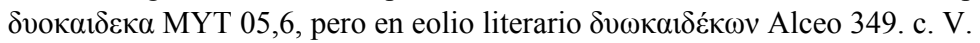

EMERITA. Revista de Lingüística y Filología Clásica (EM) - LXV 2, 1997, pp. 221-256 
M. P. Hualde Pascual - Eolismos en Jonia: Revisión de un problema de geografía intradialectal

Frente a la forma habitual del jónico-ático aicí (<*ai,ei) y su variante aćí (por yotización y caída del segundo elemento de diptongo) documentadas en las inscripciones jonias, en Quíos y Mileto aparecen formas en all M 187.11 (Mileto: ca. 450) y, en compuesto, $\alpha 1 \delta \alpha \sigma \mu$ ov SEG XXII 508.A.II.30, B.II.31 (Quíos: IV) 'sujeto a tributo perpetuo', a partir de una forma originaria *ai, $i$, (interpretable como el locativo con desinencia -i de un nombre raíz, bien como un tema en -i en «cas absolu», sin desinencia), como resultado del proceso /aywi/ > /ayyi/ > /ayi/ > /a-i/ ${ }^{97}$. Parece evidente la relación de estas formas adverbiales con las del arcadio $\alpha \mathrm{l}$ Te 4,28; $\alpha[1 I G 343.7 / 8 ; \alpha] \mathrm{l} I G 343.47 / 48^{98}$ y del eolio epigráfico (lesbio: $\alpha$ (trece ejemplos en diez inscripciones), y, en el antropónimo Aık $\lambda \varepsilon ı \delta \alpha \varsigma$ ASS 303.306.310), tesalio aıv IG IX 2.461.b.13 y beocio, con monoptongación, $\eta 1 D G E$ 6444.12.) ${ }^{99}$, presente también como $\alpha 1$ en la lírica eolia. Es evidente que las formas en ai del eolio coinciden totalmente con la forma en compuesto $\alpha \_\delta \alpha \mu$ ov de Quíos, y parcialmente con la de Mileto au (en la que el proceso habría dado un paso menos /aywi/ > /ayyi/ $>$ /ayi/ $<\alpha u>$ ), pero ello no implica su necesaria interpretación como eolismo. Una solución alternativa es considerar que las formas en $\alpha 1(1)$ suponen en jonio un arcaísmo coincidente con el eolio, al igual que con el arcadio, aunque encontramos la dificultad de que no se documentan formas de este tipo en otras áreas del jonio ni tampoco en ático ${ }^{100}$. Bien es cierto que tanto en Mileto como en Quíos se documenta en fecha posterior la forma $\alpha \varepsilon 1$

97 Para este proceso cf. E. Crespo, Elementos antiguos y modernos en la prosodia homérica, Salamanca, 1977, pp. 85-86.

98 Cf. Dubois, o. cit., p. 128-129.

99 Los datos del lesbio los tomamos de R. Hodot, o. cit. p. 72, y los del tesalio y beocio de W. Blümmel, Die aiolischen Dialekte. Phonologie und Morphologie der inschrifliche Texte aus generativer Sicht, Göttingen, 1982, p. 72. La métrica muestra que las formas en $\alpha$ son disilábicas, constituídas por dos sílabas breves, resultado del proceso /aywi/ $<62>$ /ayyi/ $<62>$ /ayi/ <62> /a-i/ (cf. Hodot loc. cit. ). El testimonio de Herodiano Gr. I,497, que da las formas $\alpha \hat{\imath}$, $\alpha \hat{\imath} 1 v$ como eolismos, no se ve confirmado por la epigrafía ni por la métrica de los textos literarios.

100 No se documentan otras formas en $\alpha(1)$ en otras zonas del jonio y para el ático epigrafico no se ha admitido la presencia del adverbio en el primer término de compuesto aıøı๐ $\varsigma$, en la que Threatte, The Grammar of Attic Inscriptions: I Phonology, Berlin-New York, 1980, pp. 276-277, cree ver un caso de itacismo, en contra de la opinión de Bechtel, Dialekte I, p. 102.

EMERITA. Revista de Lingüística y Filología Clásica (EM) - LXV 2, 1997, pp. 221-256 
M. P. Hualde Pascual - Eolismos en Jonia: Revisión de un problema de geografía intradialectal

en inscripciones dialectales: $\alpha \varepsilon 1 M 135.17$ (Mileto: a. 323), M 142.13 (Mileto: ca. 323); $\alpha \varepsilon 1$ SEG XII 390.40 (Quíos: ca. 300), en compuesto $\alpha \varepsilon \mu \nu \eta[\sigma \tau о \varsigma$ ibid. 39, si bien esto puede indicar, bien la imposición temprana de elementos de koiné, bien que el arcaísmo fue progresivamente eliminado.

\subsection{2. $\operatorname{pos}$ vs. $1 \varepsilon \rho \circ$ :}

La diversidad de formas del adjetivo que designa lo sagrado plantea un serio problema fonético y morfológico para el que, hasta la fecha, no se ha llegado a conclusiones definitivas. El hecho de que en jonio de la Dodecápolis se documenten dos formas distintas, ıро у у $є \rho \circ \varsigma$ ha llevado a pensar que la primera, coincidente con la que aparece generalizada en la vecina Lesbos, es un eolismo en jonio septentrional. La confirmación de esta hipótesis pasa por la explicación etimológica del término y de las divergencias dialectales en su vocalismo, si bien, pese a las distintas hipótesis propuestas, parece complicado sostener una forma básica única. Una propuesta reciente ${ }^{101}$ pretende la reducción de las tres formas dialectales del griego $1 \varepsilon \rho-1 \alpha \rho-1 \rho-$ a dos radicales distintos, (a) ${ }^{*} H_{l}$ eis- $/{ }^{*} H_{l} i s-$ cf. indoir. $* i s ̌$ : ai. is-; av. is- 'fuerza,

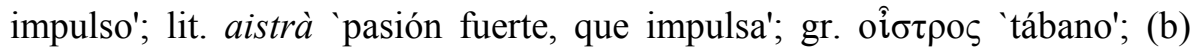
${ }^{*} H_{1} i s H_{2}$ cf. ved. isnati 'poner en movimiento'; gr. ivó $\omega$ 'evacuar'; jon. ióopal 'curar'. Para ello se basa en la diversidad de significados de i̊pós en Homero, donde la acepción de 'sagrado' en ocasiones resulta inverosímil, así en ípóv ixøv́v Il. XVI 407, referido a un pez que se agita en el anzuelo. Según esta hipótesis, ambos significados y ambas formas se habrían fundido en griego en un solo adjetivo (de ${ }^{*} H_{1}$ eis- $/ * H_{l} i s-\mathrm{y}$ de $\left.* H_{l} s H_{2}\right)$ sin que se pueda precisar cuál era la acepción originaria de cada una de las dos formas, ya que ambas habrían coincidido fonéticamente en i pós, como resultado de la evolución tanto de ${ }^{*} H_{1}$ sros como de ${ }^{*} H_{I} s H_{2}$ ros en virtud de una ley fonética (ya IE) por la que la laringal se perdería en el contexto $\mathrm{s}$ _ $\mathrm{T}$. Los dialectos griegos habrían rehecho secundariamente su forma fonética *is-ros ya en *is-arós ya en *is-eros, mientras que los dialectos que presentan i ós, con /i:/, procedente del primer alargamiento por compensación, conservarían la forma fonética como arcaísmo. La ausencia de geminación en lesbio îpos en

101 Cf. García Ramón, «ỉepós una variante, ved. isira-», VII Fachtagung der Indogermanischen Gesellschaft, Leiden, 1992, pp. 183-205.

EMERITA. Revista de Lingüística y Filología Clásica (EM) - LXV 2, 1997, pp. 221-256 
M. P. Hualde Pascual - Eolismos en Jonia: Revisión de un problema de geografía intradialectal

lugar del esperable * $\iota \rho \varsigma_{\varsigma}$ se explicaría, en ese caso, por influencia jonia. Partiendo de esta base el jonio de Asia Menor conservaría como arcaísmo formas en ipo $\varsigma$ junto a las formas propias del griego oriental rehechas en iєрos.

Sin entrar en la polémica que la formación de este término ha suscitado desde hace tiempo ${ }^{102}$ y para la que creemos que no hay de momento solución definitiva, hemos de hacer notar la acumulación mayoritaria de ipos en la zona de Jonia más septentrional y, por tanto, más cercana a la Eólide: así Quíos (5x), Eritras (4x), colonias de Focea (4x), frente a sólo cinco ejemplos en el resto de la Dodecápolis, que, por lo demás algunos son tardíos y sospechosos de influencia literaria: Samos $(2 \mathrm{x})$ y Efeso (2x), Mileto (1x). Por su parte, las formas del tipo $1 \varepsilon \rho o \varsigma$ están asentadas en toda la Dodecápolis ya desde época arcaica, si bien durante los siglos VI y V los datos se concentran en Mileto (15x), frente a un menor número de datos en las ciudades del norte de Jonia: Eritras (4x), Efeso (1x), Quíos (2x) y colonias de Focea (1x). A ello se une la presencia de un alto número de formas en $1 \rho-$, dentro del jonio insular en Tasos (8x), asimismo cercano a la Eólide, frente al resto del jonio de las islas que sólo documenta ipo ৎ en Paros (1x).

Por el contrario, en eolio de Asia las formas en ipo $\varsigma$ parecen estar perfectamente asentadas en el dialecto, contabilizándose un total de 63 formas en $1 \rho-$, a los que se añaden 41 datos de $1 \varepsilon \rho$ - con grafía itacística en inscripciones tardías, frente a sólo 16 formas en $1 \varepsilon \rho-{ }^{103}$. Es destacable, incluso, la resistencia del dialecto a la koiné en este punto, de manera que las formas en $1 \rho-$ siguen siendo mayoritarias hasta época romana.

La distribución dialectal observada en jonio y lesbio parece, en principio, avalar la interpretación de las formas en ıoৎ como eolismos en Jonia. No

102 Cf. Las distintas propuestas de los autores que operan sin laringal C. Gallavotti, «Il valore di 'hieros' in Omero e in miceneo», $A C$ 32, 1963, pp. 409-428; O. Szemerényi, «Etyma Graeca IV (22-29) Homerica at Mycenica», SMEA 20, 1979, pp. 207-211; Schwyzer Gr. Gr. I, p. 482; Lejeune, Phonétique, p. 239 n. 2; C. J. Ruijgh, «Risch, Kleine Schriften» Kratylos 29, 1984, p. 92; como de los que operan con ella M. Doria, «Testimonianze di in miceneo» Kadmos 19, 1980, p. 34; Beekes o. cit., p. 184; R. S. Schmitt, Dichtung und Dichtersprache in indogermanische Zeit, Wiesbaden, 1967, pp. 28, 112; García Ramón, art. cit., pp. 183-205.

103 Cf. Hodot, o. cit., p. 235 tab. VIII.10, tab. VIIII.11.

EMERITA. Revista de Lingüística y Filología Clásica (EM) - LXV 2, 1997, pp. 221-256 
M. P. Hualde Pascual - Eolismos en Jonia: Revisión de un problema de geografía intradialectal

obstante se plantea un serio inconveniente: si se parte para la explicación de ıрo $\varsigma$ de una protoforma *is(H)-ros, la forma $1 \rho \circ \varsigma$, con /i:/ procedente del primer alargamiento por compensación, sólo podría ser resultado fonético del jonio. La ausencia de geminación en lesbio îpos frente a un esperable **ıpos sólo puede explicarse por influencia jonia, pese a que los datos de îpoc son absolutamente mayoritarios en lesbio, frente a su carácter esporádico en jonio, y pese a lo difícil que resulta admitir que la forma supuestamente propia del lesbio $* *$ i $\rho \rho \varsigma$ haya remitido totalmente en favor del jonismo ıo $\varsigma$, sin que aparezca ni siquiera de forma residual. Lamentablemente, las dificultades etimológicas del término impiden ulteriores consideraciones. Sólo cabe decir que, si se parte de una protoforma *is(H)ros, con caída de la laringal, hemos de considerar que las formas en $1 \rho$ - son préstamos del jonio en el dialecto lesbio.

3. Reparto intradialectal: diferencias con el testimonio de Heródoto. Eolismos eliminables y eolismos confirmados. Conclusiones.

Revisados los datos, y volviendo de nuevo al mencionado pasaje de Heródoto I 142.3-4 y, por limitarnos a aquellos casos que permiten un cotejo, observamos que son grandes las inexactitudes con respecto a la división hecha por el historiador jonio:

La diptongación en el contexto de segundo alargamiento por compensación se documentaría en Quíos (supuesta modalidad eolia), pero también en Focea (supuesta modalidad lidia), en Mileto (supuesta modalidad caria) y en Tasos (fuera de la Dodecápolis); la declinación de los numerales aparece en Quíos (supuesta modalidad eolia) pero también en Samos (supuesta modalidad «pura»); supuestas geminaciones en contexto de primer alargamiento, en formas en $\Phi \alpha v v o^{\circ}$ y en $\Delta \mathrm{v} v v v^{\circ}$, se documentan en Quíos y Eritras (supuesta modalidad eolia) pero también en Teos (supuesta modalidad lidia), así como $\operatorname{ovov}(v) \eta \mu \varepsilon v \alpha$ en Samos (supuesta modalidad «pura») y en el NP $\Lambda \alpha \lambda \lambda \eta \varsigma$ de Tasos (fuera de la Dodecápolis); la palatalización en Zıovv( $\sigma \circ \varsigma)$ propia del lesbio, aparece documentada en Focea (supuesta modalidad lidia); la forma all para aıєı se atestigua en Mileto (supuesta modalidad caria) y en Quíos (supuesta modalidad eolia); hay presencia de /a:/ tras /e/, /i/ y /r/ en Quíos 
M. P. Hualde Pascual - Eolismos en Jonia: Revisión de un problema de geografía intradialectal

(supuesta modalidad eolia), pero también en Focea (supuesta modalidad lidia) y Samos (modalidad «pura»); la reducción del grupo -sthl- > -sl- aparece en Eritras y Quíos (supuesta modalidad eolia) pero también se da en Amorgos (fuera de la Dodecápolis). Sólo se correspondería exclusivamente con la modalidad eolia la pretendida declinación del participio de perfecto en *-nt- (Quíos).

Dejando, pues, de lado el testimonio de Heródoto, ¿Qué hay de cierto acerca de la presencia de elementos eolios en las ciudades de la Dodecápolis? Tras el la observación detallada de los datos de las inscripciones jonias estamos en condiciones de afirmar que algunos de estos rasgos, supuestamente compartidos por el lesbio y el jonio del Norte son claramente eliminables, en otros la cuestión del supuesto eolismo sería imprecisable, y, por último, hay confirmación de alguno de ellos como lesbismo en la variedad dialectal de determinadas ciudades de Jonia.

a. Rasgos eliminables: Podemos, a su vez, dividirlos en tres grupos:

a.1. Rasgos que han sido considerados como lesbismos por interpretación errónea de los datos:

- Participio de perfecto en *-nt-. El único dato en que se sustenta esta suposición de eolismo es la forma de Quíos $\gamma \varepsilon \gamma \omega v \varepsilon o v \tau \varepsilon \varsigma$ (s.V): Esta es, sin duda, el participio presente de un verbo, cuyo origen es el perfecto $\gamma \varepsilon \dot{\varepsilon}(\omega v \alpha$, pero que aparece ya documentado como tal presente inf. $\gamma \varepsilon \gamma \omega v \varepsilon \hat{\imath} v$ desde los poemas homéricos y, de igual manera, en las propias inscripciones arcaicas de la ciudad en cuestión, cf. también en Quíos $\gamma \varepsilon \gamma \omega v \varepsilon i ̂ v(s . V)$.

-Contracción /a:-o/ > /a:/: Descartada para el apelativo $\lambda \alpha \tau v \pi$ ov $\varsigma$ de Eritras, perfectamente asentado en la prosa jonia de Hipócrates y en otros autores. Para los antropónimos $\Lambda \alpha \mu \varepsilon \delta \omega v$ y $\Lambda \alpha \pi \rho \varepsilon \pi \eta \varsigma$ no se puede asegurar el origen jonio de los individuos designados.

a.2. Ausencia de vinculación clara del testimonio epigráfico al dialecto jonio.

-Palatalización de la dental sonora /d/ en contacto con yod: rasgo descartado, en este caso, por la debilidad del testimonio de Focea Zıvv( $\sigma \circ \varsigma)$ : se trata de un solo ejemplo, en inscripción monetaria, cuya lectura y origen no son seguros, frente a la abundancia de datos de la secuencia /di/ inicial sin palatalización, dentro y fuera del propio nombre de Dioniso, así como por el carácter tardío de los testimonios de Zovvбoৎ en Lesbio epigráfico (s.II d.C.).

a.3. Hechos que aparecen en los poetas lesbios pero ausentes en eolio epigráfico,

EMERITA. Revista de Lingüística y Filología Clásica (EM) - LXV 2, 1997, pp. 221-256 
M. P. Hualde Pascual - Eolismos en Jonia: Revisión de un problema de geografía intradialectal

posibles elementos literarios en las inscripciones jonias.

-Declinación de numerales. Hecho presente en textos epigráficos de Quíos y Samos, frente a su documentación, hasta la fecha, sólamente literaria en lesbio. Esto lleva a pensar que, mientras el hecho no se documente en textos epigráficos eolios, la flexión de los numerales podría ser un elemento literario presente en las inscripciones, más que una manifestación de la lengua hablada en la Jonia de Norte.

-Simplificación del grupo -sthl- > -sl-. Habitualmente considerado como eolismo, su documentación en lesbio se reduce, sin embargo, a los textos literarios. Por otra parte, dada la extensión del hecho por distintas áreas dialectales (arcado-chipriota, dialectos dorios, jonio) y habida cuenta de su presencia en jonio de las islas, más bien parece tratarse de un hecho propio del jonio oriental (Amorgos

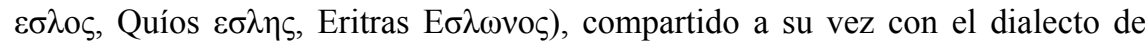
Lesbos, pero no debido a su influjo. Por otra parte, en varias ocasiones las formas en $\varepsilon \sigma \lambda$ - aparecen en textos métricos, donde no es descartable que su aparición se deba a influencia literaria.

a.4. Jonismos en lesbio.

-Tratamiento dorsal de la labiovelar en las formas tipo око, окобо et al. Como se ha puesto de relieve, hay una única forma en oкo- documentada en la Eólide frente a las formas generalizadas en о $\pi \pi 0-$. Parece ser, pues, que nos encontramos con un hecho de aislado de interferencia fonética del jonio en la vecina Eólide.

-Formas en 1 po $\varsigma$ vs. $1 \varepsilon \rho \circ \varsigma$. Si se parte de una forma previa *isros, no es posible

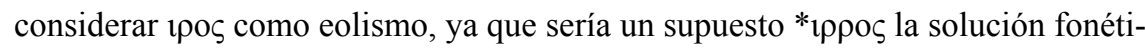
ca esperable en lesbio. Desde este punto de vista, y ante la ausencia actual de una explicación definitiva del término, parece más aconsejable entender too como préstamo jonio que se asienta en el léxico del eolio de Asia.

b. Rasgos en los que la posibilidad de eolismo resulta imprecisable.

Todos ellos supondrían arcaísmos compartidos entre el jonio y el lesbio. La debilidad de los testimonios, que en cualquier caso permiten explicaciones alternativas, impiden determinar en qué medida las formas mencionadas presentan o no una geminación eolia:

-Estadio de geminación en el contexto de primer alargamiento por compensación:

(a) las formas con geminación presentes en las series de antropónimos en $\Phi \alpha v v 0^{\circ}$ y $\Delta{\mathrm{v} v v v^{\circ}}^{\circ}$, supuestamente creadas sobre los radicales *phauesn- y

EMERITA. Revista de Lingüística y Filología Clásica (EM) - LXV 2, 1997, pp. 221-256 
M. P. Hualde Pascual - Eolismos en Jonia: Revisión de un problema de geografía intradialectal

*duis-nu-, son débiles testimonios de una supuesta geminación eolia por las siguientes razones: (i) la etimología de las mismas es difícil de establecer de forma definitiva; (ii) los antropónimos son una clase de palabras con menor fuerza testimonial para demostrar en ellos la presencia de eolismos, por su carácter de términos viajeros que pueden pasar de un territorio a otro por una simple cuestión de moda; (iii) nos encontramos con NP compuestos o claramente hipocorísticos y podemos suponer que en ellos el grupo $/ \mathrm{nn} /$ no es la continuación de un antiguo /sn/, sino un caso de geminación expresiva, muy frecuente tanto en frontera de compuesto como en las formas de hipocorístico. También para la forma de Tasos $\Lambda \alpha \lambda \lambda \eta \varsigma$ puede excluirse la posibilidad de eolismo, si entendemos que se trata de un radical de origen indígena con la consabida geminación expresiva.

(b) Las supuestas formas de orónimo "A tan en textos literarios, lo que las hace débiles como testimonios dialectales.

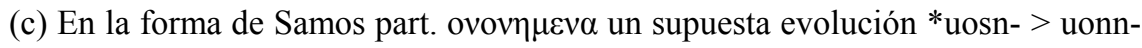
no puede ser corroborada por las supuestas deficiencias gráficas del texto, sin contar, además, la propia complicación morfológica de un participio que presenta una formación evidentemente analógica. En cualquier caso, de aceptarse la supo-

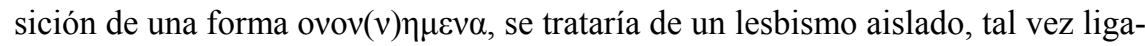
do a un préstamo léxico en un verbo de uso frecuente entre hablantes de ambos dialectos, pero en ningún caso se puede decir que sea un hecho que se haya integrado en el sistema fonético del jonio.

-Permanencia de /a:/ tras /e/,/i/ o /r/: La forma $\theta \varepsilon \alpha \varsigma$ (Antípolis: 450-425) documentada en las colonias puede dejar sentir la influencia de la lengua homérica.

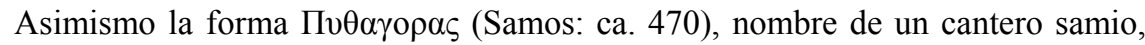
documentado en una inscripción de Olimpia, puede estar influída por el dialecto

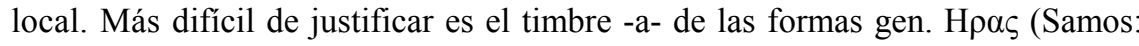

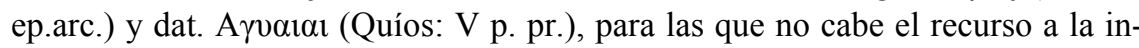
fluencia externa. Unicamente cabría considerarlas como aticismos tempranos, pero resulta sorprendente, aunque no imposible, que el prestigio del ático haya impuesto su vocalismo al nombre de divinidades de arraigada tradición local.

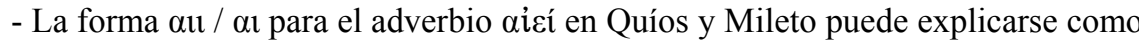
un arcaísmo compartido entre el jonio y el eolio, conservado en esta dos ciudades de forma residual. Sin embargo no puede descartarse definitivamente su interpretación como eolismo en Jonia, desde el momento en que no se documentan formas en $\alpha 1(l)$ en otras áreas del jonio.

EMERITA. Revista de Lingüística y Filología Clásica (EM) - LXV 2, 1997, pp. 221-256 
M. P. Hualde Pascual - Eolismos en Jonia: Revisión de un problema de geografía intradialectal

c. Eolismos confirmados en Asia Menor.

Se trata de préstamos presentes en algunas ciudades jonias de Asia (Quíos, Mileto), de las islas (Tasos) y en las colonias de Focea (Ampurias) y se producen en el terreno de la morfología, dos de ellos en formas desinenciales:

- El más claro eolismo confirmado como tal es la forma de $3^{\mathrm{a}} \mathrm{pl}$. en - $\omega \iota \mathrm{l} /$ - -oıбl en las ciudades de Quíos, subj. $\lambda \alpha \beta \omega \imath \iota v, \pi \rho \eta \xi o ı \sigma ı v(V)$, colonias de Focea subj.

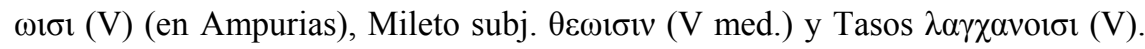
Como se ha puesto de relieve recientemente ${ }^{104}$, la presencia de estos eolismos presupone que la diptongación en este contexto en Jonia no es un proceso fonético, como prueba el cumplimiento del alargamiento compensatorio en una misma inscripción (cf. ac. pl. $\tau o \varsigma$ ac.pl. $\tau \alpha \varsigma \chi \omega \rho \alpha \varsigma$ ), sino influencia del dialecto de la Eólide sobre el territorio vecino.

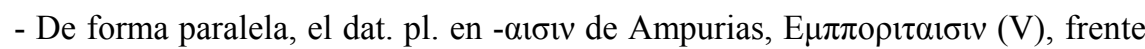

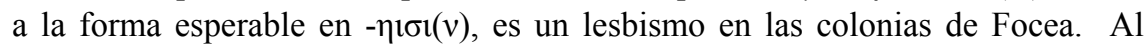
igual que en el rasgo anterior, la conservación de la /a:/ sin anteriorización no sería tanto un hecho fonético como el préstamo de una marca morfológica del eolio al jonio de Asia.

- La forma part. dat. $\delta \iota \psi \alpha v \tau[\imath$ de Ampurias puede suponer asimismo un eolismo

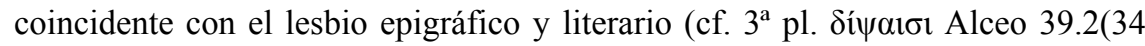
D.)) en cuanto a su conjugación atemática correspondiente a lo que sería un verbo contracto esperable en jonio.

Recapitulando lo dicho destacamos las siguientes conclusiones:

(1) Se confirma la presencia del elemento eolio en jonio oriental, pero en menos casos de lo que habitualmente se consideraba, al descartar datos que se habían interpretado de forma errónea y otros cuya vinculación dialectal no es clara.

(2) Los eolismos confirmados en Jonia son producto de la situación de bilingüismo producida en Asia Menor por el contacto entre los hablantes de los dialectos lesbio y jonio. Esto posibilita la influencia en ambos sentidos y la aparición de préstamos e interferencias, en cualquier caso, de lesbismos en jonio y de jonismos en lesbio. Se debe rechazar, por tanto, la explicación de los eolismos como producto del sustrato en ciudades a las que se atribuye un

104 Cf. Hernández Vázquez, art. cit. p. 289.

EMERITA. Revista de Lingüística y Filología Clásica (EM) - LXV 2, 1997, pp. 221-256 
M. P. Hualde Pascual - Eolismos en Jonia: Revisión de un problema de geografía intradialectal

origen eolio.

(3) Nos encontramos ante préstamos de carácter morfológico: la dipton-

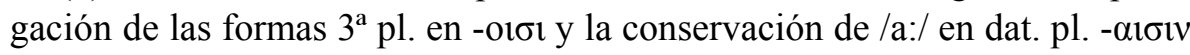
no obedecen a procesos fonéticos, sino que son simples préstamos de marca morfológica.

(4) Los mencionados préstamos se producen de manera esporádica, siempre en inscripciones arcaicas, y hay contraejemplos documentados incluso

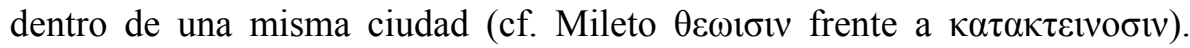
Dentro de las restricciones que nos impone lo reducido del material epigráfico de cada ciudad anterior al siglo V, la propia dispersión geográfica de Jonia y sus colonias, la temprana aparición de aticismos y la pronta extensión de la koiné en jonio, podemos afirmar, dado el carácter esporádico de estos préstamos y los contraejemplos documentados, que no son formas que lleguen a integrarse plenamente dentro del sistema lingüístico del jonio.

MARÍA Pilar Hualde Pascual 